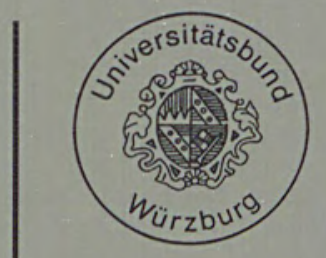

Würzburger Vorträge zur Rechtsphilosophie, Rechtstheorie und Rechtssoziologie

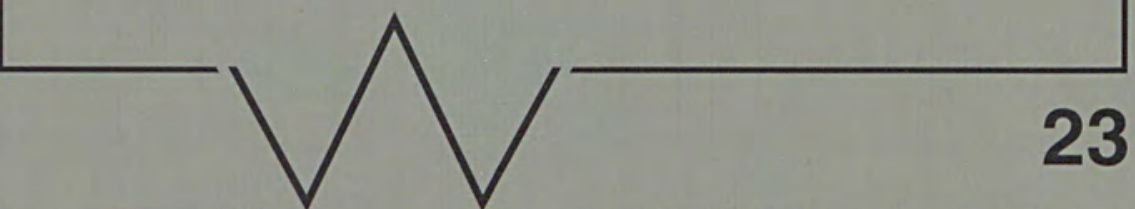

Gertrude Lübbe-Wolff

Recht und Moral im Umweltschutz 



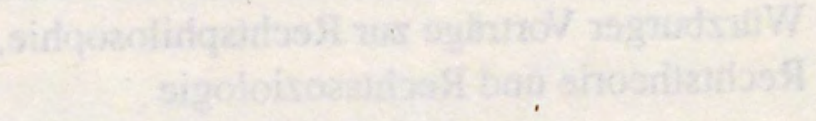

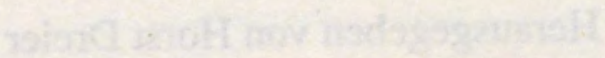

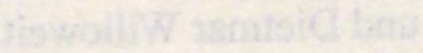

$\cos 215$ 
Würzburger Vorträge zur Rechtsphilosophie, Rechtstheorie und Rechtssoziologie

Herausgegeben von Horst Dreier und Dietmar Willoweit

Begründet von Hasso Hofmann, Ulrich Weber und Edgar Michael Wenz †

\section{Heft 23}




\section{Gertrude Lübbe-Wolff}

\section{Recht und Moral \\ im Umweltschutz}

Nomos Verlagsgesellschaft Baden-Baden 
Vortrag gehalten am 15. Februar 1998

Die Deutsche Bibliothek - CIP-Einheitsaufnahme

\section{Lübbe-Wolff, Gertrude:}

Recht und Moral im Umweltschutz / Gertrude Lübbe-Wolff. - 1. Aufl. Baden-Baden : Nomos Verl.-Ges., 1999.

(Würzburger Vorträge zur Rechtsphilosophie, Rechtstheorie und Rechtssoziologie ; H. 23) ISBN 3-7890-5644-8

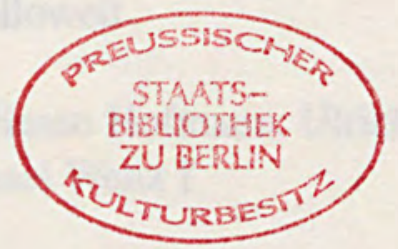

1. Auflage 1999

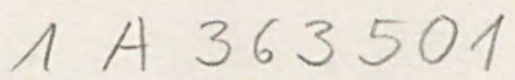

(ㄷ) Nomos Verlagsgesellschaft, Baden-Baden 1999. Printed in Germany. Alle Rechte, auch die des Nachdrucks von Auszügen, der photomechanischen Wiedergabe und der Übersetzung, vorbehalten. Gedruckt auf alterungsbeständigem Papier. 


\section{Inhaltsverzeichnis}

1. Worum es gehen soll 6

2. Recht, Moral und Öko-Philosophie 8

3. Recht, Moral und Eigenverantwortung

- die Deregulierungswelle als Moralisierungsschub?

4. Moral statt Recht?

a) Chancen des Umweltschutzes über rechtsfreie Umweltmoral 18

b) Warum nicht mehr moralische Virtuosen? 24

c) Der Mensch - ein moralisches Mängelwesen? 26

d) Institutionelle Kultur 28

5. Möglichst viel moralische Selbststeuerung, möglichst wenig rechtliche Fremdsteuerung?

a) Recht als Amoralisierungsfaktor? 33

a) Bedenken 33

bb) Moral ermöglichende und Moral unterstützende Funktionen des Rechts $\quad 40$

cc) Moralbedarf für die Konstitution vernünftigen Rechts

b) Recht, Moral und Freiheit 44

6. Schluß 


\section{Worum es gehen soll}

Die Unterscheidung zwischen Recht und Moral ist in der Geschichte der Rechts- und Moralphilosophie für unterschiedliche Zwecke konzipiert, und das Verhältnis von Recht und Moral ist auf unterschiedliche Weise bestimmt und in unterschiedlichen Hinsichten problematisiert worden ${ }^{1}$. Mit der Überschrift „Recht und Moral im Umweltschutz" ist deshalb noch nicht sehr genau bezeichnet, worum es gehen soll. In der jüngeren Vergangenheit, nach dem Ende des zweiten Weltkriegs und der nationalsozialistischen Diktatur, hat man sich für das Verhältnis von Recht und Moral vor allem unter dem Gesichtspunkt des Kollisionsfalles interessiert. Es ging um die Frage, wie begrifflich und praktisch mit Widersprüchen zwischen den Anforderungen des positiven Rechts und denen der Moral umzugehen ist: Kann und darf kraß unmoralisches positives Recht noch „Recht“ genannt, muß oder darf ihm noch Gehorsam geleistet werden? Schützt solches Recht diejenigen, die ihm gemäß gehandelt haben, vor moralischer Verurteilung und vor nachträglicher rechtlicher Sanktionierung durch ein anderes Rechtssystem ${ }^{2}$ Der durch die Wiedervereinigung ausgelöste Bedarf

1 Näher dazu Gertrude Lübbe-Wolff, Historische Funktionen der Unterscheidung von Recht und Moral, Archiv für Rechts- und Sozialphilosophie, Beiheft 23, 1985, S. $43 \mathrm{ff}$.

2 Nachweise bei Lübbe-Wolff(Fn. 1), S. 43; aus jüngerer Zeit s. Robert Alexy, Begriff und Geltung des Rechts, 1992; Joachim Lege, Pragmatik und Jurisprudenz (Habilitationsschrift, erscheint demnächst), Typoskript S. 571 ff., jew. m.w.N. Dieselben Fragen wurden und werden auch in anderer Terminologie, als Fragen nach dem Verhältnis von Recht und Gerechtigkeit (s. Gustav Radbruch, Gesetzliches Unrecht und übergesetzliches Recht, in: ders., Der Mensch im Recht. Ausgewählte Vorträge und Aufsätze über Grundfragen des Rechts, 1957, S. 111 ff., 118 ff. [zuerst erschienen in SJZ 1946, S. 105 ff.]) oder positivem Recht und Naturrecht, gestellt. Natürlich bedeuten „Gerechtigkeit" und „Naturrecht" nicht dasselbe wie „Moral", und angesichts der zwischen diesen Begriffen bestehenden Bedeutungsunterschiede bringt wohl am präzisesten die Formulierung des angesprochenen Kollisionsproblems als eines Problems der Kollision von Recht und Gerechtigkeit auf den Punkt, worum es geht. Von den bestehenden Bedeutungsunterschieden waren aber die praktischen Erkenntnisinteressen der mit diesen unterschiedlichen Begriffen geführten Diskussion des Kollisionsproblems kaum affiziert, so daß sich cum grano salis doch behaupten läßt, die in diesen unterschiedlichen terminologischen Fassungen diskutierten Fragen seien ein und dieselben gewesen. 
an juristischer Vergangenheitsbewältigung hat die Diskussion dieser Fragen, vor allem im Zusammenhang mit den Mauerschützenprozessen, reaktiviert ${ }^{3}$.

Die Überlegungen, die ich im folgenden zum Verhältnis von Recht und Moral im Umweltschutz anstellen möchte, haben einen ganz anderen Gegenstand. Sie betreffen die Frage, inwieweit für die Lösung von Umweltproblemen einerseits auf positives Recht, andererseits auf Moral zu setzen ist. Die Frage, die behandelt und deren Aktualität gleich noch demonstriert werden soll, ist also, mit anderen Worten, die nach dem richtigen Einsatzbereich einerseits von positivem Recht, andererseits von Moral, wenn es um die richtige Orientierung umweltrelevanten Verhaltens geht.

Indem ich die Frage so stelle, will ich selbstverständlich nicht unterstellen, daß Moral ein Steuerungsinstrument ist, über das irgendwelche zentralen Entscheidungsinstanzen in ähnlicher Weise verfügen und das sie in ähnlicher Weise zum Einsatz zu bringen oder nicht zum Einsatz zu bringen beschließen könnten wie die Steuerungsinstrumentarien des positiven Rechts. Auch wenn Moral, als verhaltenslenkende Ressource betrachtet, sehr viel weniger disponibel, vor allem sehr viel weniger für zentrale Steuerungsinstanzen gezielt disponibel ist als positives Recht ${ }^{4}$, hat es doch Sinn, das Verhältnis von Recht und Moral einmal

3 Horst Dreier, Gustav Radbruch und die Mauerschützen, JZ 1997, S. 421 ff.; Gerhard Sprenger, 50 Jahre Radbruchsche Formel oder: Von der Sprachnot der Juristen, NJ 1997, S. 3 ff.; Walter Gropp, Naturrecht oder Rückwirkungsverbot? Zur Strafbarkeit der Berliner „Mauerschützen“, NJ 1996, S. 393 ff.; Arthur Kaufmann, Die Radbruchsche Formel vom gesetzlichen Unrecht und vom übergesetzlichen Recht in der Diskussion um das im Namen der DDR begangene Unrecht, NJW 1995, S. 81 ff.; Ralf Dreier, Juristische Vergangenheitsbewältigung, 1995, S. 28 ff.; Frank Salinger, Radbruchsche Formel und Rechtsstaat, 1995; Helmut Lecheler, Unrecht in Gesetzesform? Gedanken zur „Radbruch'schen Formel“, 1994; Robert Alexy, Mauerschützen: zum Verhältnis von Recht, Moral und Strafbarkeit, 1993; Knut Amelung, Strafbarkeit von „Mauerschützen“ - BGH, NJW 1993, 411, JuS 1993, S. 637 ff.; Monika Frommel, Die Mauerschützenprozesse - eine unerwartete Aktualität derRadbruch'schen Formel, in: FS für Arthur Kaufmann, 1993, S. 81 ff.

4 Nach verbreiteter Auffassung liegt die Erzeugung moralischer Grundsätze und Antriebe sogar völlig außerhalb der staatlichen Einflußsphäre, s. etwa, unter Berufung auf Georg Jellineks Allgemeine Staatslehre (2. Aufl. 1905, S. 243), Klaus M. Leisinger, 
als ein Verhältnis verhaltenslenkender Ressourcen zueinander ins Auge zu fassen. Zumindest über die Reichweite der Steuerung umweltrelevanten Verhaltens durch positives Recht wird ja in einer Gesellschaft positiv entschieden, und die insoweit zu treffenden Entscheidungen darüber, was wie zum Gegenstand rechtlicher Regelung gemacht wird und was nicht, werden unter anderem auf Annahmen über die Chancen, Nachteile und Vorzugswürdigkeiten moralischer Selbststeuerung als Alternative $\mathrm{zu}$ rechtlicher Regulierung des betreffenden Sachbereichs gestützt. Was für Überzeugungen sollte man also diesbezüglich vernünftigerweise haben?

\section{Recht, Moral und Öko-Philosophie}

Wenn man bedenkt, daß die Moralphilosophie sich in Europa über Jahrhunderte in engem Zusammenhang mit der Rechtsphilosophie und schließlich gerade in wechselseitiger Abgrenzung zu dieser entwickelt hat, ist es bemerkenswert, daß das Recht, und insbesondere die uns hier interessierende Frage des Verhältnisses von Recht und Moral, in den Erzeugnissen der praktischen Umweltphilosophie, insbesondere der deutschen praktischen Umweltphilosophie, so gut wie keine Rolle spielt. Der Markt ist überschwemmt mit Publikationen zur Umweltphilosophie, Öko-Philosophie, Umweltethik, Öko-Ethik, Naturethik usw. Die Forschungstätigkeit in diesem Bereich hat ein solches Ausmaß angenommen, daß man von einer neuen Teildisziplin der Philosophie sprechen kann ${ }^{5}$. Die Frage, in welchem Verhältnis die Umweltethik

Unternehmensethik, 1997, S. 42. Mit dieser Auffassung ist aber das komplexe Wechselspiel von staatlichem Recht und durch staatliches Recht geprägten Institutionen einerseits und bürgerschaftlicher Moral andererseits nicht zureichend erfaßt.

5 Vgl. nur an neueren deutschsprachigen Veröffentlichungen Angelika Krebs (Hrsg.), Naturethik, 1997; dies., Ökologische Ethik I: Grundlagen und Grundbegriffe, in: Julian Nida-Rümelin (Hrsg.), Angewandte Ethik, 1996, S. 346 ff.; Anton Leist, Ökologische Ethik II: Gerechtigkeit, Ökonomie, Politik, ebda. S. 386 ff;; Dieter Birnbacher (Hrsg.), Ökophilosophie, 1997; Klaus Michael Meyer-Abich, Praktische Naturphilosophie, 1997; Andreas Brenner, Ökologie-Ethik, 1996, jew. m.w.N.; für die US-amerikanische Umweltphilosophie s. neben den zahlreichen Beiträgen ihrer 
zum Umweltrecht steht, kommt dabei aber kaum vor; relativ selten findet man hier das Recht, die geltende rechtliche Ordnung unseres Verhältnisses zur Natur und die bestehenden Möglichkeiten der Steuerung umweltrelevanten Verhaltens durch Recht, auch nur erwähnt ${ }^{6}$.

Die Umweltethik hat sich in die Fragen verbissen, deren juristisches Analogon im Zusammenhang mit Art. 20a GG unter den Stichworten „Anthropozentrik“" versus „Ökozentrik“ diskutiert wird: Hat die Natur moralischen Eigenwert? Und: Kann es Pflichten auch gegenüber der nicht menschlichen Natur, ja sogar gegenüber der unbelebten Natur geben? Mit der vorherrschenden Fixierung auf diese Fragen und einer verbreiteten, pragmatisch funktionslosen Fixierung auf das For-

Vertreter in den o.a. deutschsprachigen Sammelwerken Robin Attfield, Environmental Philosophy: Principles and Prospects, 1994; als Klassiker der ÖkoPhilosophie s. Arne Naess, The Shallow and the Deep, Long-Range Ecology Movement. A Summary, Inquiry 16 (1973), S. 95 ff. (s. auch ders., Ecology, community and lifestyle, 1989); John Passmore, Man's Responsibility for Nature. Ecological Problems and Western Traditions, 1974; Hans Jonas, Das Prinzip Verantwortung. Versuch einer Ethik für die technologische Zivilisation, 1979 (im folgenden zitiert nach der stw-Ausgabe, 1. Aufl. 1984). Für einen instruktiven, kritischkonstruktiven Überblick s. Konrad Ott, Ökologie und Ethik, 2. Aufl. 1994.

6 S. aber als Versuch einer Zusammenführung der beteiligten Disziplinen Julian Nida-Rümelin/Dietmar v.d. Pfordten (Hrsg.), Ökologische Ethik und Rechtstheorie, 1995; die hier versammelten Beiträge bleiben allerdings in auffälliger Weise auf das Lieblingsthema der Umweltethik, die Anthropozentrismus-Frage, konzentriert (dazu sogleich im Text). Eingang in die umweltethische Literatur hat im übrigen noch am ehesten die praktisch vergleichsweise unwichtige, mit der Einfügung des Art. 20a in das Grundgesetz hierzulande vorläufig abgeschlossene Diskussion über eine Verankerung des Umweltschutzes in der Verfassung gefunden, s. etwa Ott (Fn. 5), S. 141 ff., mit zutreffender skeptischer Einschätzung der Wirkungsmöglichkeiten eines „Grundrechts auf Natur". Zu den Gründen der Wirkungsschwäche und daher auch sehr begrenzten praktischen Relevanz verfassungsrechtlicher Umweltschutzgebote (umweltbezogener Grundrechte, Staatszielbestimmungen u.a.) s. Gertrude LübbeWolff, Zielorientierte Umweltpolitik - Probleme der rechtlichen Steuerung, in: Friedrich Hinterberger/ Andreas Renner (Hrsg.), Zukunftsfähigkeit durch Wettbewerb, 1998 (im Erscheinen). Zur verbreiteteren Diskussion von Gerechtigkeitsfragen - z.B. Fragen der internationalen und intergenerationellen Gerechtigkeit - im Zusammenhang mit der Nutzung von Umweltgütern s. statt vieler Avner de-Shalit, Why Posterity Matters, 1995; Otfried Höffe, Moral als Preis der Moderne, 3. Aufl. 1995, S. 172 ff;; Leist (Fn. 5), S. 388 ff., m.w.N. 
schungsziel ,zwingender" Beweisführung (möglichst aus einem Prinzip!) bleibt die Umweltphilosophie in dem größten Teil ihrer Erzeugnisse nicht nur steril für die Frage, die uns hier interessieren soll, sondern verwendet überhaupt viel Scharfsinn in einer geradezu gespenstischen Distanz zu allem, was konkret gedacht und getan werden muß, um zu einem vernünftigeren Umgang mit der Natur zu finden. Zu dieser Einschätzung paßt die Beobachtung, daß die Erzeugnisse der ÖkoEthik außerhalb der einschlägigen Gemeinde keine Resonanz finden: „,... like Hume's Treatise, they fall deadborn from the press. " ${ }^{7}$ Und anders als im Falle von Hume's Treatise, so bliebe hinzuzufügen, sind auch die Perspektiven für eine spätere rezeptionelle Auferstehung nicht gut.

Was konkrete Orientierungsleistungen für Umweltpolitik oder individuelles Umweltverhalten angeht, führt die ambitionierteste unter den Neuerscheinungen der deutschen Öko-Philosophie zu Erkenntnissen wie der, daß das Fällen eines Baumes nicht bedingungslos verboten, sondern unter Umständen auch erlaubt sei, dies aber nur unter der Voraussetzung, daß für die Fällung so gute Gründe sprechen, daß der Fäller, sich in die Rolle des Baumes versetzend - nämlich unter der Prämisse, daß er selbst als Baum wiedergeboren werden könnte - der Fällung nicht würde widersprechen wollen ${ }^{8}$. Solche Ergebnisse vielhundertseitiger Bemühungen sind als philosophischer Beitrag zu der praktischen Frage, wie wir uns in Ansehung der Natur richtig verhalten, zutiefst inadäquat. Warum? Erstens, weil etwaige Streitfragen, die die ethische Erlaubtheit des Baumfällens betreffen, für alle praktisch relevanten Streitfälle mit der aufgestellten Regel nicht geklärt, sondern

nur auf die Frage der angemessenen Aufopferungsbereitschaft von

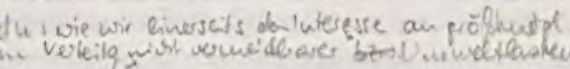

7 Andrew Light/Eric Katz, Introduction, in: dies. (Hrsg.), Environmental Pragmatism, 1996, S. 1. Zur Kritik philosophischer Ethiken des „leeren Sollens“ allgemeiner nicht nur bezogen auf die Öko-Ethik - Uwe Gerecke, Soziale Ordnung in der modernen Gesellschaft. Ökonomik - Systemtheorie - Ethik, 1998 (im Erscheinen), S. $294 \mathrm{ff}$. und passim. Für Ansätze zur Selbstkritik in der - hier verallgemeinernd als „akademische Ethik" angesprochenen - philosophischen Ethik Gernot Böhme, Ethik im Kontext. Über den Umgang mit ernsten Fragen, 1997, S. $11 \mathrm{ff}$.

8 Meyer-Abich(Fn. 5), S. 367. 
Bäumen, wenn es um ihre eigene Fällung geht, verlagert werden. Zweitens, und das ist der wichtigere Punkt, weil die Behandlung des Baumfällungsthemas als eine Frage der ethischen Erlaubtheit individueller Baumfällaktionen uns das große einschlägige Umweltproblem, nämlich die Abholzung der tropischen Regenwälder', implizit als ein Problem präsentiert, das seine wesentliche Ursache in ethischen Fehleinstellungen, in fehlender Einsicht in den moralischen Eigenwert der Bäume, hat, und dementsprechend durch Korrektur dieser Fehleinstellungen gelöst werden muß. Damit wären wir beim Thema. Die Behauptung, daß diese Unterstellung inadäquat ist und auf dieser Inadäquanz die spontan empfundene Lächerlichkeit des zitierten Baumschutzprinzips beruht, wird im folgenden noch näher zu begründen sein.

\section{Recht, Moral und Eigenverantwortung - die Deregulierungswelle als Moralisierungsschub?}

Man kann sagen, daß seit Beginn der 90er Jahre das Verhältnis von Recht und Moral ein zentrales umweltpolitisches Diskussionsthema ist. Die Rede ist hier von der Diskussion über Deregulierung, also den Abbau von Rechtsvorschriften und durch Rechtsvorschriften bedingten ökonomischen Belastungen. Daß die Deregulierungsdiskussion im Kern eine Diskussion über das Verhältnis von Recht und Moral im Umweltschutz ist, fält allerdings nicht ohne weiteres auf, denn das Wort „Moral" wird in dieser politischen Diskussion selten verwendet. Der Schlüsselbegriff, mit dem in der Deregulierungsdiskussion das bezeichnet wird, was an die Stelle der abzubauenden und abgebauten Rechtsvorschriften und behördlichen Kontrollen treten soll, lautet vielmehr: Eigenverantwortung. Das seit Beginn der 70er Jahre beständig ausgebaute Umweltordnungsrecht, so heißt es, sei zu umfangreich,

9 Dazu Katrina Brown/David W. Pearce (Hrsg.), The Causes of Tropical Deforestation, 1994; Torsten Amelung, Globaler Umweltschutz als Verteilungsproblem im Nord-Süd-Konflikt, 1997, S. 22 ff. 
zu anforderungsreich, zu kompliziert geworden, es produziere mehr Bürokratie als Umweltschutz, sei in der Praxis ohnehin nicht mehr vollziehbar, habe sich zu einem Standortnachteil im globalisierten Wettbewerb ausgewachsen und müsse deshalb zugunsten von mehr umweltbezogener Eigenverantwortung der Unternehmen zurückgefahren werden ${ }^{10}$. Worum handelt es sich aber bei der unternehmerischen Eigenverantwortung, die hier offenbar als funktionales Äquivalent für die reduzierte staatliche Steuerung durch Umweltrecht und dessen behördliche Überwachung gehandelt wird?

Aus der Etymologie des Wortes „verantworten“ wird häufig abgeleitet, daß Verantwortung etwas mit der Pflicht, zu antworten, mit Rechenschaftspflichtigkeit, zu tun hat. Dieses Bedeutungselement steckt besonders deutlich im Begriff der Verantwortlichkeit. Verantwortlich ist man jemandem, und die Verantwortlichkeit diesem ,jemand" gegenüber besteht zuallererst darin, daß man ihm Rechenschaft über dasjenige Verhalten schuldet, in bezug auf das man verantwortlich ist. In dieser Rechenschaftspflicht erschöpft sich aber Verantwortlichkeit nicht.

Jede Verantwortlichkeit impliziert, daß derjenige, demgegenüber Rechenschaft abgelegt wird, im Prinzip in der Lage ist, aus der Art und Weise, in der Rechenschaft abgelegt wurde, Konsequenzen zu ziehen, die den Rechenschaftspflichtigen betreffen. So bezeichnẹn wir den Kanzler oder Ministerpräsidenten einer parlamentarischen Regierung als dem Parlament gegenüber verantwortlich, weil er dem Parlament nicht nur Rechenschaft über sein Regierungsverhalten abzulegen hat, sondern auf der Grundlage der gegebenen Rechenschaft vom Parlament auch abgewählt (oder zumindest: nicht wiedergewählt) werden kann. Der Abteilungsleiter, der der Unternehmensleitung gegenüber

10 Die Vorstellung, daß dabei jedem Rückzug des Staates ohne weiteres ein entsprechender Zuwachs an unternehmerischer Eigenverantwortung korrespondiert, hat nach einem gerade veröffentlichten Artikel meiner Lokalzeitung auf besonders schöne und schlichte Weise die Vizepräsidentin der Handwerkskammer Ostwestfalen-Lippe in einer Rede zum Ausdruck gebracht: „Weniger Gebote und Verbote würden die Eigenverantwortlichkeit der Unternehmen stärken", Neue Westfälische v. 27.1.1998, Artikel „Handwerk ökologischer". 
für einen bestimmten Geschäftsbereich verantwortlich ist, hat dieser nicht nur Rechenschaft über die Entwicklung dieses Geschäftsbereichs abzulegen, sondern auch damit zu rechnen, daß sie aus der erfolgten Rechnungslegung gegebenenfalls Konsequenzen zieht, die sein Gehalt oder sogar seine Position betreffen. Wären die Dinge so organisiert, $\mathrm{da} ß$ der Abteilungsleiter lachend mit beliebigen Eröffnungen über Mißwirtschaft und Verluste auftreten könnte, ohne für sich die geringsten negativen Folgen befürchten zu müssen, würden wir es ablehnen, hier überhaupt noch von Verantwortlichkeit zu sprechen, oder zumindest festhalten wollen, daß es sich hier nicht mehr um richtige, wirkliche Verantwortlichkeit handelt, und daß hier auch nicht mehr Verantwortung im eigentlichen Sinne des Wortes getragen wird.

Im Konsequenzen-tragen-Müssen scheint danach ein wesentliches Bedeutungselement des Verantwortungsbegriffs zu liegen ${ }^{11}$. Das Element der Rechenschaftspflicht spielt demgegenüber nur eine dienende Rolle, insofern es nämlich überall dort zur Verantwortung gehört, wo die zu tragenden Konsequenzen vom Willen eines Dritten abhängen, der über das zu verantwortende Verhalten und/oder dessen Ergebnisse ins Bild gesetzt werden muß, um Konsequenzen daran knüpfen zu können. Dementsprechend verwenden wir den Verantwortungsbegriff auch in Zusammenhängen, in denen ersichtlich keine spezifischen Rechenschaftspflichten impliziert sind, wohl aber Konsequenzen getragen werden müssen. In diesem Sinne kann, ohne daß das Wort unpassend wirkt, beispielsweise Eltern Erziehungsverantwortung für ihre Kinder zugeschrieben oder einem jungen Erwachsenen, wenn er volljährig wird, gesagt werden, daß er von nun an seine Lebensführung selbst zu verantworten habe. Die Rede von der elterlichen Erziehungsverant-

11 In diesem Sinne auch Garrett Hardin, The Tragedy of the Commons, Science 162 (1968), S. 1243 (1247); ebenso im Zusammenhang mit der aktuellen umweltpolitischen „Eigenverantwortungs"-Diskussion Franz-Josef Moormann, Staatliche Überwachung und Eigenverantwortung der Unternehmen im Lichte der EGUmweltaudit-Verordnung und des deutschen Umweltauditgesetzes, in: Ministerium fir Umwelt, Raumordnung und Landwirtschaft des Landes Nordrhein-Westfalen (Hrsg.), Neue Entwicklungen im Umweltrecht (Tagungsband der Umweltrechtstage 1996), S. 83 ff. (85). Für die ganz andere Verwendung des Verantwortungsbegriffs bei Hans Jonas s. noch unten Text mit Fn. 14. 
wortung verweist nicht auf irgendeine institutionalisierte Rechenschaftspflicht, sondern darauf, daß neben den Kindern im Zweifel die Eltern selbst die ersten sind, die die Folgen vernachlässigter oder falsch wahrgenommener Erziehungsaufgaben in Gestalt unfreundlicher, rücksichtsloser, disziplinfreier oder sonstwie nicht ihren positiven Möglichkeiten entsprechend geratener Kinder zu ertragen haben. Und von der Entlassung eines erwachsen gewordenen Menschen in die Selbstverantwortung kann deshalb angemessen die Rede sein, weil an die Stelle elterlichen Schutzes vor allem, was man früher noch nicht selbst „verantworten“ konnte, nun das Lebenmüssen mit den Konsequenzen des eigenen Verhaltens tritt.

Wenn in der Deregulierungsdiskussion postuliert wird, daß mehr Eigenverantwortung der Umweltnutzer an die Stelle rechtlicher Überregulierung treten solle, wird der Verantwortungsbegriff aber nicht in diesem Sinne gebraucht. Zwar gibt es Bereiche, in denen unsachgerechtes umweltrelevantes Verhalten auch für den Verursacher unangenehme Konsequenzen hat. So etwa, wenn aufgrund unzureichender Einweisung des Bedienungspersonals in einem Chemiewerk ein Druckkessel explodiert und dadurch gefährliche Substanzen freigesetzt werden: Sachschaden, Produktionsausfall, vielleicht sogar Personenschäden, Verunsicherung und Empörung in der Belegschaft und in der Nachbarschaft, Imageverlust in der breiteren Öffentlichkeit, verstärkte Aufmerksamkeit der Behörden - lauter Unliebsamkeiten also sind die Folge. In vielen anderen Bereichen sind aber mit einem mehr oder weniger sorgfältigen, mehr oder weniger korrekten, mehr oder weniger umweltschonenden Verhalten solche unmittelbar die Handelnden selbst treffenden Konsequenzen nicht verbunden. Wer auf der grünen Wiese baut statt auf einem sanierten innerstädtischen Altstandort, hat keinen persönlichen Nachteil davon, daß er die mit höherem Naturverbrauch verbundene Alternative gewählt hat. Wer bei Abwassereinleitungen oder Abluftemissionen Vorsorgegrenzwerte überschreitet, verursacht in aller Regel keine sichtbaren Schäden, die ihm individuell zurechenbar wären, sondern trägt nur auf unmerkliche Weise zur Steigerung der allgemeinen Schadstoffbelastung von Wasser, Luft und Boden bei. Und wer problematische Flüssigabfälle gelegentlich fein 
dosiert über die Kanalisation entsorgt oder durch unsorgfältigen Umgang mit wassergefährdenden Stoffen solche Stoffe in das Grundwasser gelangen läßt, hat wenig Grund zu der Befürchtung, daß die Konsequenzen solchen Verhaltens ihn unmittelbar tangieren oder ihm über Entdeckung und Zurechnung als von ihm verursacht mittelbar angelastet werden, wenn nicht Voraussetzungen dafür durch spezifische geeignete Kontrollmechanismen geschaffen sind. Wenn hier nun dereguliert oder Deregulierung gefordert wird, man also beispielsweise das Bauen im Außenbereich oder die planerische Umwandlung von Außenbereichs- in Bauflächen erleichtert, behördliche Überwachungsmaßnahmen einschränkt oder sogar bereichsweise ausschließt, Anlagenbetreiber aus der Verpflichtung entläßt, sich über Art, Anzahl und Zustand ihrer Anlagen zum Umgang mit wassergefährdenden Stoffen durch Aufstellung eines entsprechenden Katasters Rechenschaft abzulegen, usw., dann bleiben keine Mechanismen der Folgenanlastung übrig, und es entstehen auch keine neu, mit Bezug auf die hier von Verantwortung im oben erläuterten Sinne gesprochen werden könnte ${ }^{12}$.

Wenn in der Praxis nichtsdestoweniger Politik mit der Formel gemacht wird, daß an die Stelle detaillierter behördlicher Genehmigungsprüfungen, immissionsschutzrechtlicher Regelüberwachung, behördlicher Kontrollen im Abfallnachweisverfahren usw. ,mehr Eigenverantwortung" der umweltrelevant Handelnden treten solle, so werden wir damit auf nicht weniger und nicht mehr als das Pflichtbewnßtsein, die Moral der von rechtlicher Regelung und behördlicher Überwachung zu entlastenden Wirtschaftssubjekte verwiesen ${ }^{13}$. Dies ist der rein mo-

12 Dies gilt unter bestimmten Voraussetzungen, die die Wirtschaftsverbände beharrlich ansteuern, auch für den Sonderfall der Deregulierung im Zusammenhang mit dem Öko-Audit, s. dazu noch unten, Text mit Fn. 15.

13 Soweit es dabei um Entlastung nicht von materiellrechtlichen Anforderungen, sondern von Prüfungs- und Überwachungsmaßnahmen geht, die der Kontrolle der Einhaltung materiellrechtlicher Anforderungen dienen, ist Rechtlichkeit oder Rechtschaffenheit die Tugend, auf die damit verwiesen wird: Statt auf behördliche Fremdkontrolle soll man sich auf unterstellte Bereitschaft der Betriebe verlassen, selbst für die Umweltrechtskonformität des eigenen Verhaltens zu sorgen. 
ralisch-appellative Verantwortungsbegriff, der im Anschluß an Hans Jonas endemisch geworden ist ${ }^{14}$.

Soviel zur Begründung der These, daß die politische Diskussion über umweltrechtliche Deregulierung und Eigenverantwortung, wie sie seit einigen Jahren geführt wird, der Sache nach eine Diskussion über das Verhältnis von Recht und Moral im Umweltschutz ist. Im Ergebnis hat diese Diskussion bereits zu erheblichen Terrainverlusten für das Umweltrecht und die typischen Instrumente seiner Durchsetzung geführt.

Lassen Sie mich die Rolle, die die Moral in dieser Diskussion spielt, noch an zwei Beispielen aus Diskussionsveranstaltungen illustrieren. Im einen Fall waren in einem Vortrag unter anderem bestimmte Deregulierungsmaßnahmen und andere Rechtsänderungen kritisiert worden, die die behördliche Überwachung umweltrelevanter Aktivitäten teils rechtlich einschränken, teils, indem sie anderen behördlichen Aufgaben Priorität zuweisen, faktisch ausschließen. Begründet worden war diese Kritik mit der Prognose, daß die Vermehrung der kontrollfreien Räume und Zeiten zu einer Vermehrung der Gesetzesverstöße, also zu einer Verschärfung des altbekannten Problems der Vollzugsdefizite

14 Hans Jonas, Das Prinzip Verantwortung. Versuch einer Ethik für die technologische Zivilisation (zuerst 1979), Ausgabe Frankfurt a.M. 1984. Das Spezifikum der moralischen Verpflichtung, die Jonas als Verantwortung bezeichnet, liegt nur in ihrem Gegenstand, nämlich darin, daß sie sich auf das Gedeihen eines der eigenen Macht unterworfenen Lebendigen bezieht, s. Jonas, aaO. S. $172 \mathrm{ff}$. Für die zwischenzeitliche Verbreitung dieses extensiven, meist dann auch nicht mehr mit der bei Jonas vorgesehenen gegenständlichen Beschränkung verwendeten Verantwortungsbegriffs s. statt vieler Walther Ch. Zimmerli, Wandelt sich die Verantwortung mit dem technischen Wandel? in: Hans Lenk/Günter Ropohl (Hrsg.), Technik und Ethik, 1987, S. 92 ff.; Ernst-Joachim Lampe (Hrsg.), Verantwortlichkeit und Recht, 1989; Carl Friedrich Gethmann/Michael Kloepfer/Hans G. Nutzinger, Langzeitverantwortung im Umweltstaat, 1993; Kurt Bayertz, Eine kurze Geschichte der Herkunft der Verantwortung, in: ders., (Hrsg.), Verantwortung. Prinzip oder Problem?, 1995, S. 3 ff. (Bayertz, aaO., S. 5 u. passim, charakterisiert als „Verantwortung" die Zurechnung der von einem Subjekt kausal bewirkten Handlungsfolgen, faßt damit aber den Verantwortungsbegriff keineswegs enger als Hans Jonas, dessen Verantwortungsbegriff ja ebenfalls eine (moralische) Zurechnung der Folgen nicht ausreichender Verantwortungswahrnehmung impliziert - nur impliziert er eben nicht, daß diese Folgenzurechnung auch irgendwelche Folgen hat; deshalb trägt man an dieser Form der „Verantwortung“ so leicht). 
einschließlich der damit verbundenen auch ordnungspolitisch bedenklichen Ungerechtigkeiten, führen würde. In der sich anschließenden Diskussion rief das unter anderem einen Redner auf den Plan, der deutlich mißgestimmt beklagte, wie sehr die Diskussion von Mißtrauen gegenüber der Wirtschaft geprägt sei; in diesem Klima könne man nicht $\mathrm{zu}$ vernünftigen Lösungen kommen. Noch deutlicher fiel die Mißbilligung in einem anderen Fall aus. In diesem Fall hatte ich mich gegen einen bayerischen Deregulierungsvorschlag gewandt, der darauf hinauslief, daß Betriebe, die am EG-Öko-Audit-System teilnehmen, von den Immissionsschutzbehörden nicht mehr routinemäßig überwacht werden dürfen. Eine solche Regelung, so hatte ich argumentiert, sei durch die vorausgesetzte Beteiligung am Öko-Audit-System - einer Art Selbstkontrollsystem, an dem auch ein vom jeweiligen Unternehmen beauftragter unabhängiger Gutachter beteiligt ist - nicht gerechtfertigt, sondern werde im Gegenteil die Funktionsfähigkeit dieses Systems gefährden, weil durch den Wegfall der behördlichen Überwachung der praktisch wichtigste völlig betreiberunabhängige Kontrollmechanismus wegfalle und auf diese Weise der externe Gutachter einen wichtigen Rückhalt für die Integrität seiner Prüfungen verliere, indem er sich eventuellen Zumutungen des Betreibers, fünfe gerade sein zu lassen, nicht mehr mit Verweis auf die Gefahr behördlicher Entdekkung entziehen könne ${ }^{15}$. Von dieser Überlegung zeigte sich ein anwesender Unternehmerverbandsvorsitzender so empört, als sei er höchstpersönlich beleidigt worden: Wenn mit solchen Unterstellungen gearbeitet werde, weigere er sich, noch weiter in diesem Raum zu diskutieren; dies müsse er sich nicht bieten lassen.

Wo lag hier jeweils das Problem? Das Problem lag darin, daß die zitierten Argumente für die Beibehaltung bestimmter behördlicher Überwachungsmaßnahmen von der Unterstellung Gebrauch gemacht hatten, Wirtschaftssubjekte könnten durch den Wegfall dieser Überwa-

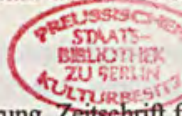

15 Näher dazu Gertrude Lübbe-Wolff, Öko-Audit und Deregulierung, Zeitsehrift für Umweltrecht 1996, S. 173 ff. Der kritisierte Deregulienungsvorschlag ist in Bayern schließlich nicht umgesetzt worden; eine entsprechende Regelung wurde stattdessen in Niedersachsen getroffen. 
chungsmaßnahmen veranlaßt werden, es mit der Einhaltung der geltenden Regeln weniger genau zu nehmen und in diesem Sinne auch Einfluß auf die von ihnen bestellten Gutachter auszuüben. Der Stein des Anstoßes bestand also darin, daß man von der Möglichkeit unmoralischen Verhaltens ausgegangen war. Dies wurde im einen Fall als unsachgerecht und betrüblich, im anderen Fall als seinerseits unmoralisch, nämlich als ungerechtfertigter Angriff auf die Ehre eines Berufsstandes und seiner Mitglieder, zurückgewiesen. Daß diese Art und Weise, beanspruchte Moralität gegen das Recht und die spezifischen Instrumente seiner Kontrolle und Durchsetzung in Stellung zu bringen, nicht etwa Ausdruck besonders hoher moralischer Standards, sondern Ausdruck eines in Deutschland leider verbreiteten Mangels an institutioneller Kultur ist, werde ich noch zu begründen versuchen.

\section{Moral statt Recht?}

\section{a) Chancen des Umweltschutzes über rechtsfreie Umweltmoral}

Was können wir nun tatsächlich im Hinblick auf den notwendigen Schutz der Umwelt von moralischen Anstrengungen erwarten?

Es bedarf keiner großen Bemühungen, um zu erkennen, daß man auf rein moralisch motivierte, nicht durch Recht flankierte Verhaltensänderungen zugunsten der Umwelt keine großen Hoffnungen setzen sollte. Das gilt unabhängig davon, ob wir von einem sehr engen Begriff des Moralischen ausgehen und etwa mit Kant oder Schopenhauer als moralisch nur Verhaltensweisen anerkennen, deren ausschließliche Triebfeder die Pflicht ist $(\text { Kant })^{16}$ oder die vollkommen frei von jedem egoistischen Beweggrund sind (Schopenhauer) ${ }^{17}$, oder ob wir von ei-

16 Immanuel Kant, Die Metaphysik der Sitten (1797), A 14, 15. S. dagegen für die Annahme, daß Pflichtbewußtsein zwar (anders als Vernunft) ein Motiv sein kann, aber nicht dasjenige ist, was eine Handlung zu einer tugendhaften macht, David Hume, A Treatise of Human Nature (1739/40), Buch II, Teil III, Abschn. III und Buch III, Teil II, Abschn. I.

17 Arthur Schopenhauer, Preisschrift über die Grundlage der Moral (1841), in: ders., 
nem weiteren Moralbegriff ausgehen, der als durch Moral gesteuert jedes Verhalten insoweit ansehen würde, als es durch die Aussicht auf damit $\mathrm{zu}$ erwerbende Achtung oder Mißachtung bestimmt ist $(\text { Luhmann })^{18}$. Daß wir für die Lösung von Umweltproblemen nicht ohne Recht auskommen können, ist im Grunde trivial. Es lohnt sich aber, sich die Gründe für diesen trivialen Sachverhalt vor Augen zu führen, weil das den Sinn für das Ausmaß schärft, in dem rechtliche Lösungen tatsächlich erforderlich sind.

Die schlechten Aussichten für ,rein moralische" Problemlösungen hängen mit dem Charakter der Umweltmedien - der Luft, des größten Teils der Gewässer und in gewissen Hinsichten sogar des Bodens - als „commons" zusammen, d.h. als Gemeinschaftsgüter oder Kollektivgüter, bei denen übermäßige individuelle Nutzungen (d.h. Nutzungen, die den individuellen Anteil an der schadlos möglichen Gesamtnutzung überziehen) nicht in spezifischer Weise den exzessiven individuellen Nutzer, sondern die Gesamtheit der Nutzer belasten, individuelle Nutzungsverzichte nicht in spezifischer Weise dem Individuum, sondern der Allgemeinheit der Nutzer zugute kommen, und schließlich analog asymmetrische Kosten-Nutzen-Verhältnisse auch in bezug auf

Werke in zehn Bänden (Zürcher Ausgabe), Band VI, 1977, S. 143 ff. (243 ff.: Abwesenheit jedes egoistischen Motivs als Kriterium der im positiven Sinne moralischen Qualität, d.h. des moralischen Werts einer Handlung); Schopenhauer identifiziert als einzigen nichtegoistischen Antrieb und damit als Quelle aller Moralität das Mitleid. Vgl. dagegen Hutcheson, der ebenfalls die Abwesenheit jedes selbstsüchtigen Motivs zum Kriterium der Tugendhaftigkeit einer Handlung bestimmt, das Mitleid (die Mitempfindung, sympathy) aber, qua Übernahme von Gefühlen anderer als eigene, noch nicht als selbstlosen Antrieb gelten lassen will, sondern eine vom Mit-Leiden unabhängige Fähigkeit zu moralischer Wahrnehmung (,moral sense“) und moralischen Willensakten (,benevolence“) annimmt: Francis Hutcheson, A System of Moral Philosophy, Zwei Bände, 1755, Nachdruck New York 1968, Bd. I, S. 6 ff. ( 43,47 ff.).

18 S. Niklas Luhmann, Ökologische Kommunikation, 3. Aufl. 1990, S. 259 f., zu Moral als einer „Konditionierung von Achtungs- oder Mißachtungszuteilungen“. Zuordnungsobjekte für das Adjektiv ,moralisch" sind hier nicht Handlungen, sondern Kommunikation und deren Codierung; die Anwendung des Moralbegriffs auf Verhalten und die dem angepaßte Erläuterung oben im Text nimmt sich insofern eine übersetzerische Freiheit heraus. 
eventuelle zur Erhaltung oder Steigerung der Nutzbarkeit erforderliche Investitionen bestehen ${ }^{19}$. Jeder weiß, daß der Aufbau und die Aufrechterhaltung einer Moral, die die exzessive Nutzung solcher Gemeinschaftsgüter verhindert, schon in kleinsten Sozialverbänden hohe Anforderungen an alle Beteiligten stellt. Man muß nur an die gemeinsame Nutzung eines Glases Nutella durch mehrere Geschwister denken. Selbst unter den für Aufbau und Erhaltung einer gemeinsamen Moral günstigen Verhältnissen eines kleinen Verbandes mit hoher Interaktionsdichte treten häufig Symptome moralischer Überforderung auf, die dann zur Erfindung von Institutionen zur Absenkung des Moralbedarfs führen - beispielsweise dazu, daß das Nutellaglas von den Eltern unter Verschluß genommen wird und nur noch zum Frühstück auf den Tisch kommt, wo die Nutzung unter unmittelbarer sozialer Kontrolle erfolgt.

Je größer die Anzahl der Personen, die ein Gemeinschaftsgut gemeinsam nutzt, desto unwahrscheinlicher wird es, daß die vernünftige Nutzung und Pflege des betreffenden Gemeinschaftsgutes ohne ein durch rechtliche Regeln institutionalisiertes System äußerer Anreize zu sachgerechtem Verhalten, allein durch eine gemeinsame Moral, erreicht werden kann. Moral kann nämlich nur als gemeinsame aufgebaut und erhalten werden. Selbst ein elementarer Antrieb für moralisches Verhalten wie das Mitleid, das einige Philosophen sogar für die einzige

19 Für die Insuffizienz moralischer Appelle zum Schutz von Gemeinschaftsgütern allg. Hardin (Fn. 11), S. 264 und passim; speziell für den Bereich des Umweltschutzes Bruno S. Frey, Ökonomie ist Sozialwissenschaft, 1990, S. 37 ff.; Guy Kirsch, Umweltmoral - Ein Ersatz für staatliche Umweltpolitik?, in: Klaus Mackscheidt/Dieter Ewringmann/Erik Gawel (Hrsg.), Umweltpolitik mit hoheitlichen Zwangsabgaben? 1994, S. 261 ff.; Gertrud Nunner-Winkler, Moral und Politik - Eine Frage des Systems oder der Persönlichkeit?, in: Hans-Ulrich Derlien/Ute Gerhardt/Fritz W. Scharpf (Hrsg.), Systemrationalität und Partialinteresse, Festschrift für Renate Mayntz, 1994, S. 123 ff., 141. Für die Möglichkeit selbstorganisierter Institutionalisierung von Regelsystemen für die Nutzung von Gemeinschaftsgütern (und für eine institutionalisierte Sanktionierung von Regelverstößen als wesentliche Erfolgsvoraussetzung in den analysierten Experimentalfällen!) Elinor Ostrom/James Walker/Roy Gardner, Covenants with and without a Sword: Self-Governance is Possible, American Political Science Review.86 (1992), S. 404 ff. 
Quelle aller Moral gehalten haben ${ }^{20}$, verkümmert und kann in sein Gegenteil, in Lust am Leiden anderer, verkehrt werden, wo er nicht als ein gemeinsamer erfahren wird. Und jeder von uns hat wohl selbst die Erfahrung gemacht, daß es für die moralischen Grundsätze, denen wir uns verpflichtet fühlen, oder genauer: für dieses Gefühl der Verpflichtung und die Bereitschaft, sich dementsprechend zu verhalten, nichts Abträglicheres gibt als die Entdeckung oder, vorsichtiger ausgedrückt, die Entstehung des Eindrucks, daß der Respekt für diese Grundsätze von den meisten anderen nicht geteilt wird oder man sogar ganz allein damit steht ${ }^{21}$. Ulrich Wickerts Buchtitel „Der Ehrliche ist der Dumme" spielt auf eine problematische Weise mit diesem Tatbestand. Es könnte sein, daß Herr Wickert allein mit der Wahl dieses Titels mehr Schaden für die öffentliche Moral angerichtet hat, als er durch den sehr viel weniger bekannten Inhalt des Buches wiedergutmachen kann.

Mit der Befolgung moralischer Grundsätze, die gravierende Verzichtleistungen außerhalb eines Zusammenhangs gesicherter Gegenseitigkeit bzw. Verallgemeinerung erfordern, sind Menschen im allgemeinen überfordert. Nehmen wir das Beispiel des Autofahrens. Wir alle wissen, daß der motorisierte Individualverkehr schon in seinem gegenwärtigen Ausmaß, und erst recht wenn wir die Partizipationsansprüche von mehreren Milliarden weiteren potentiellen Verkehrsteilnehmern in den noch wenig motorisierten Ländern berücksichtigen,

20 Schopenhauer (Fn. 17), S. 248 u. passim; für die - nicht auf das Mitleid beschränkte Mitempfindung (sympathy) als Quelle der Moral Adam Smith, The Theory of Moral Sentiments (1759), Erster Teil; vgl. auch Hutcheson (Fn. 17). Zur Geschichte der Mitleidsethik und ihrer Rolle in den aktuellen Diskussionen über moralische Verpflichtungen im Verhältnis zur nichtmenschlichen Umwelt Ott (Fn. 5), S. 144 ff.

21 S. speziell zur Abhängigkeit umweltgerechten Verhaltens vom Vertrauen darauf, daß auch andere sich umweltgerecht verhalten, Hans Spada, Umweltbewußtsein: Einstellung und Verhalten, in: Lenelis Kruse/Carl-Friedrich Graumann/ErnstDieter Lantermann (Hrsg.), Ökologische Psychologie, 1990, S. 623 ff. (628 f.), m.w.N. Für daraus folgenden Bedarf an umweltbezogenen Regulierungen, die „die moralisch Handelnden nicht länger als die ,Dummen' dastehen" lassen, auch Vittorio Hösle, Moral und Politik. Grundlagen einer politischen Ethik für das 21. Jahrhundert, 1997, S. 1103. 
auf eine Übernutzung globaler Umweltressourcen hinausläuft. Für sehr viele Menschen, die an diesem motorisierten Individualverkehr altersoder einkommensbedingt oder aus sonstigen Gründen nicht oder nur sehr eingeschränkt teilnehmen können, und die deshalb nicht an seinen Vorteilen, wohl aber an seinen zahlreichen Nachteilen partizipieren, bedeutet er außerdem eine ganz akute, schwere Beeinträchtigung ihrer Lebensqualität. Viele von uns werden sich deshalb schon einmal gefragt haben, ob man unter diesen Bedingungen nicht eigentlich moralisch verpflichtet wäre, auf Besitz und regelmäßige Nutzung eines Kraftfahrzeuges zu verzichten. Was blüht aber demjenigen, der eine solche Verpflichtung für sich bejaht und befolgt? Da es keinerlei Gewähr dafür gibt, daß andere sich genauso verhalten werden, muß er damit rechnen, bei Verzicht auf alle Annehmlichkeiten des motorisierten Individualverkehrs - Komfort, Zeitersparnis, höhere Mobilität, dadurch ermöglichte höhere Erlebnisdichte und erleichterte Wahrnehmung aller möglichen Chancen - doch weiterhin von dessen sämtlichen Nachteilen behelligt zu bleiben: schlechte Luft zu atmen, auf fast allen Wegen und womöglich sogar in der eigenen Wohnung von Lärm verfolgt zu sein, Grau statt Grün zu sehen, kleinere Kinder nicht unbeaufsichtigt und ungefährdet aus dem Haus lassen zu können, und so weiter. Unter diesen Umständen eine individuelle Entscheidung gegen das Autofahren, oder für eine Reduktion der individuellen Kfz-Nutzung auf ein menschheitsverträgliches Niveau, zu treffen, überfordert die durchschnittliche moralische Leistungsfähigkeit.

Erst recht ist nicht zu erwarten, daß irgendeine Moral, irgendein die natürliche Umwelt betreffendes Wertbewußtsein den brasilianischen Bauern, dessen Felder ausgelaugt sind und ihn nicht mehr ernähren, davon wird abhalten können, neues Land für sich urbar zu machen, indem er ein Stück Wald abbrennt ${ }^{22}$. Es ist im Gegenteil so gut wie ausgeschlossen, daß Menschen unter den Lebensbedingungen eines sol-

22 Für den erheblichen und wachsenden Anteil der so motivierten Waldzerstörung am Problem der Tropenentwaldung s. David Pearce/Katrina Brown, The issues, in: dies. (Fn. 9), S. 32 f.; Amelung (Fn. 9), 54 ff. 
chen brasilianischen Bauern sich eine Moral zu eigen machen, die ihnen die Waldrodung verbietet.

Und natürlich sehen die Chancen für rein moralische individuelle Selbststeuerung auch nicht besser aus, wenn wir statt des Bauern, der von der Subsistenzwirtschaft lebt, Akteure ins Auge fassen, die nicht für sich selbst, sondern für den Markt produzieren. Die Entscheidungsträger in einer Ledergerberei oder im Chemiewerk wissen im Zweifel, daß die Chromgerbung des Leders und die Chlororganika, die das Chemiewerk verkauft, Umweltprobleme verursachen. Der Markt ist aber nun einmal ein System, das die Vermeidung von Umweltproblemen nicht eo ipso honoriert, sondern im Gegenteil denjenigen mit Konkurs bedroht, der an der Nachfrage vorbei produziert oder vorhandene Nachfrage weniger gut bedient als die Konkurrenz. Wer unter diesen Umständen aus ökologischen Gründen darauf verzichtet, eine vorhandene Nachfrage nach umweltschädlichen Chlororganika oder umweltschädlich mit Chromgerbung hergestelltem Leder zu bedienen, nimmt sich damit nur selbst vom Markt und überläßt das Feld der mit weniger Skrupeln beladenen Konkurrenz. Die Erfolgsaussichten für moralisch motiviertes Handeln sind insofern auf dem Angebotsmarkt tendenziell noch ungünstiger als in der Sphäre des Konsumverhaltens. Während man als Konsument durch die Entscheidung, nicht Auto zu fahren, der Umwelt doch immerhin unter Umständen ein kleines Quantum an Belastungen ersparen $\mathrm{kann}^{23}$, würde die Entscheidung des Chemieproduzenten, eine nachgefragte problematische Chemikalie nicht mehr anzubieten, nicht die Umwelt von dieser Chemikalie entlasten, sondern nur das entsprechende Marktsegment anderen überlassen. Das bedeutet nicht, daß es in der Wirtschaft keine Spielräume

23 Das gilt z.B., wenn in ländlichen Gegenden ohne Stau- und Parkplatzsorgen auf den Gebrauch eines Autos verzichtet wird. Unter Großstadtbedingungen, d.h. unter Bedingungen knapper Fahrmöglichkeiten, läßt sich dagegen die Sache auch so betrachten, daß individueller Fahrverzicht durch die damit verbundene Entlastung des Parkraums und der Straßen zur rush-hour nur den Freiraum zum Fahren für andere erhöht bzw. die für andere bestehenden negativen Anreize reduziert: es werden sozusagen nur Parklücken für andere frei gemacht, s. Helmut Wiesenthal, Ökologischer Konsum - ein Allgemeininteresse ohne Mobilisierungskraft?, in: Eckart Hildebrandt (Hrsg.), Ökologischer Konsum, 1990, S. 21 ff. 
für ökologisch oder gar überhaupt für moralisch orientiertes Handeln gäbe $^{24}$. Es bedeutet aber, daß es unsinnig wäre, eine Lösung konsumund produktionsbedingter Umweltprobleme unabhängig von institutionellen Lösungen, die die Lasten der erwünschten Rücksichtnahme auf die Umwelt wettbewerbsmäßig neutralisieren, von individuellen „moralischen“" Entscheidungen der Wirtschaftssubjekte zu erwarten.

$\mathrm{Da}$ man hier faktisch Problemlösungen allein über Moral nicht erwarten kann, ist, wie schon gesagt, im Grunde trivial - so trivial wie die Feststellung, daß wir aus ganz ähnlichen Gründen, die schon Hob$b_{e s}{ }^{25}$ analysiert hat, auch hinsichtlich der meisten anderen Verhaltenserwartungen, von deren Befolgung die gute Ordnung einer Gesellschaft abhängt, nicht ohne eine Stabilisierung dieser Verhaltenserwartungen durch Recht auskommen. So trivial also wie die Einsicht, daß wir nicht nur eine Vertragsmoral, sondern auch ein Vertragsrecht, nicht nur eine Familienmoral, sondern auch ein Familienrecht, nicht nur eine Steuermoral, sondern auch ein Steuerrecht brauchen. Bevor ich $\mathrm{zu}$ - leider offenbar nicht ganz trivialen - Schlußfolgerungen aus dem Bisherigen komme, noch zwei Bemerkungen zu der von mir behaupteten Überforderung moralischer Leistungsfähigkeit in den angeführten Beispielsfällen.

\section{b) Warum nicht mehr moralische Virtuosen?}

Erstens: Ich habe von einer Überforderung der durchschnittlichen moralischen Leistungsfähigkeit gesprochen. Ist diese durchschnittliche Leistungsfähigkeit aber nicht vielleicht steigerbar? Größere moralische Leistungen liegen ja nicht prinzipiell außerhalb menschlicher Möglichkeiten. $\mathrm{Zu}$ allen Zeiten hat es Menschen gegeben, deren moralische

24 Zur Diskussion über die Existenz solcher Spielräume in der Wirtschaftswissenschaft s. Horst Steinmann/Birgit Gerhard, Effizienz und Ethik in der Unternehmensführung, in: Karl Homann (Hrsg.), Aktuelle Probleme der Wirtschaftsethik, 1992, S. 159 ff.; Jean Paul Thommen, Betrachtungen zum Verhältnis zwischen Betriebswirtschaftslehre und Unternehmensethik, in: Hans G. Nutzinger (Hrsg.), Wirtschaftsethische Perspektiven III, 1996, S. 173 ff. (189 ff.).

25 Thomas Hobbes, Leviathan (1651), s. insbes. Teil II (Of Commonwealth). 
Leistungsfähigkeit die ihrer Mitmenschen weit außerhalb der üblichen Schwankungsbreiten überragte. In vielen religiösen Traditionen, zum Beispiel im Katholizismus, werden solche Menschen als Heilige verehrt. Zeigt die Existenz solcher Individuen nicht, daß die Menschheit zu Besserem als dem oben zugrundegelegten moralischen Normalmaß fähig ist? Beispielsweise gibt es ja auch Menschen, die aus ökologischer Überzeugung - nicht nur wegen ohnehin geringen Bedarfs oder ohnehin fehlender Mittel, sondern unter erheblichem freiwilligem Verzicht - auf den Besitz eines eigenen Autos verzichten und auch sonst für den Umweltschutz überdurchschnittliche Opfer bringen. Muß man nicht darauf hoffen und alles daransetzen, daß mehr, daß alle so werden wie diese? Man sollte bei allem, was Menschen betrifft, sehr vorsichtig mit der Behauptung sein, irgendetwas sei völlig ausgeschlossen. Alle Erfahrung spricht aber dagegen, daß die moralische Leistungsfähigkeit von Individuen in beliebiger Anzahl in diesem Ausmaß steigerbar ist. Vermutlich liegt das daran, daß auch ,moralische Virtuosen" $(\text { Max Weber })^{26}$ eine Gratifikation benötigen, um zu Virtuosen werden und Virtuosen bleiben zu können, und daß diese Gratifikation in Gestalt von sozialer Achtung, Selbstachtung, Heilsgewißheit, oder was sonst ins Spiel kommen mag, je nach Grad der Virtuosität nur hoch genug ausfällt, wenn der Virtuose einzigartig oder doch zumindest der Kreis oder die Gruppe der Virtuosen begrenzt und abgegrenzt bleibt. Was den moralischen Virtuosen auf den ersten Blick zum Rätsel macht, wäre danach gerade die Bedingung seiner Möglichkeit. Der moralische Virtuose kann das leisten, was er leistet, nicht obwohl, sondern weil die anderen nicht dasselbe leisten.

26 Zum religiösen (moralischen) Virtuosentum, zu dessen christlicher Ausprägung als auf Armut, Keuschheit und Gehorsam gegründetes Mönchtum und zur Ablösung der diesbezüglichen ethischen Ideale durch den Protestantismus Max Weber, Die protestantische Ethik I, 4. Aufl. 1975, S. 318 ff. 
Zum zweiten die behauptete Überforderung betreffenden Punkt. Von einer „Überforderung der durchschnittlichen moralischen Leistungsfähigkeit" zu sprechen, hat einen Nachteil: Man könnte dies als Feststellung eines Defizits auffassen und einen Unterton des Bewertens und Bedauerns heraushören. So ist die getroffene Feststellung aber nicht gemeint. Gerade in dem Mangel an instinktiver Festlegung des Menschen auf sozialverträgliches Verhalten - genauer: in den Fähigkeiten, die mit diesem Mangel kompensatorisch verbunden sind - liegen ja sehr viel reichhaltigere Problemlösungsoptionen, als den bedingungslos instinktiv zu sozialverträglichem Verhalten disponierten Lebewesen zur Verfügung stehen ${ }^{27}$.

Wir mögen kein Talent zu bedingungsloser Orientierung unseres individuellen Verhaltens an verallgemeinerbaren Grundsätzen haben. Dafür sind wir aber in der Lage, im verabredeten Zusammenwirken mit anderen die Rahmenbedingungen unseres Verhaltens selbst zu organisieren. Wir haben, mit anderen Worten, das Talent, Institutionen zu bilden, in denen auf der Grundlage von Gegenseitigkeitsbeziehungen sozialverträgliches Handeln ohne Überforderung moralischer Leistungsfähigkeit ermöglicht und wahrscheinlich gemacht wird. Dieses Talent bietet mehr Möglichkeiten als eine verläßlichere, bedingungslosere Disposition zum Je-für-sich-richtig-Handeln böte. Gerade weil wir nicht disponiert sind, bedingungslos jedes Verhalten einfach einzustellen, dessen Maxime wir als nicht verallgemeinerbar erkannt haben, gerade weil wie also beispielsweise nicht disponiert sind, aus bloBer Einsicht individuell je für uns unsere Kfz-abhängige Mobilität im erforderlichen Ausmaß, nehmen wir einmal an um $80 \%$, zu reduzieren, können wir institutionelle Arrangements erfinden, die auf sehr viel intelligentere Lösungen hinauslaufen als auf einen achtzigprozentigen

27 Zur generell schwachen Instinktbindung und ihren Korrelaten als Besonderheiten der menschlichen Konstitution allg. Arnold Gehlen, Der Mensch, 8. Aufl. 1962. Mit Gehlens hier (S. 392 ff.) eingebrachter Theorie elementarer Institutionenbildung hat allerdings das oben Ausgeführte nichts zu tun. 
Mobilitätsverzicht für jedermann bei sonst gleichbleibenden Bedingungen.

Wenn wir es als eine Art Fehlkonstruktion der menschlichen Natur betrachten wollten, daß unsere moralische Leistungsfähigkeit in der oben beschriebenen Weise begrenzt ist, mißbilligten wir also eine wesentliche Voraussetzung für den Reichtum der Möglichkeiten, durch den sich das Sozialleben der Menschen von dem der Tiere unterscheidet und auf dem der bisherige evolutionäre Erfolg der Gattung Mensch beruht. Auf diese (oder auf andere) Weise die Natur des Menschen an moralischen Grundsätzen zu messen und für nicht genügend $\mathrm{zu}$ befinden, hat im übrigen, da solche Kritik nicht zu einer Verbesserung ihrer Objekte führen kann, ohnehin keinen $\operatorname{Sinn}^{28}$. Umgekehrt müssen postulierte moralische Werte und Normen sich daran messen lassen, ob sie der Natur des Menschen gerecht werden ${ }^{29}$.

28 Zur Kritik solcher ,moralischer" Kritik der menschlichen Natur vgl. Baruch Spinoza, Ethica (1677), Vorwort zum Dritten Teil.

29 Diese Überzeugung ist, ganz besonders im Lande Kants, der die ältere Tradition des engen Zusammenhangs der Ethik mit der Anthropologie beendet hat, alles andere als eine Selbstverständlichkeit und bedürfte insofern ausführlicherer Begründung. An dieser Stelle muß ich mich auf den Hinweis beschränken, daß auch Ethiken, in deren Begründung Postulate der Angepaßtheit an menschliche Natur und Lebensbedingungen keine Rolle spielen oder sogar explizit zurückgewiesen werden, de facto regelmäßig Merkmale solcher Anpassung aufweisen (oder, um es neutraler auszudrücken, Merkmale aufweisen, die als Ergebnis solcher Anpassung gedeutet werden könnten). So sind z.B. in der katholischen Morallehre die Grenzen allgemeinmenschlicher Leistungsfähigkeit berücksichtigt mit der Unterscheidung zwischen praecepta (Geboten) und consilia (Räten, d.h. Angeratenem, dessen Befolgung verdienstlich, dessen Nichtbefolgung aber keine Sünde ist), s. zu dieser Unterscheidung Manfred Scheuer, Die evangelischen Räte, 2. Aufl. 1992; Johannes Gründel, Art. Consilia Evangelica, in: Theologische Realenzyklopädie, Bd. VIII, 1. Aufl. 1981, S. 192 ff.; konziser Franz Lau, Art. Evangelische Räte, in: Die Religion in Geschichte und Gegenwart, Bd. 2, 3. Aufl. 1958, Sp. 785 ff. Eine säkularisierte Parallele hat diese Berücksichtigung menschlicher Verhaltensdispositionen und Empfindlichkeiten in der rechts- und moralphilosophisch allgemein üblichen tradierten Unterscheidung und differenzierten Behandlung von negativen (Nichtschädigungs-) und positiven (Wohltätigkeits-) Pflichten. Für die - konfusionsfreie - Behauptung eines Zusammenhangs zwischen moralischen Geltungsansprüchen und empirischen Realisationsbedingungen auch Gerecke (Fn. 7), S. 291 ff. 


\section{d) Institutionelle Kultur}

Für die Moral des Umgangs mit Gemeinschaftsgütern heißt das: moralisch geboten sind nicht der aufopferungsvolle, d.h. der große Nutzungsverzicht oder die aufopferungsvolle Pflegeinvestition, die unabhängig vom Verhalten aller anderen geleistet werden; moralische "Gebote" dieses Inhalts zielen an den faktischen Möglichkeiten der Problemlösung vorbei und könnten, wenn sie für verbindlich gehalten würden, nur Überforderungsspannung, fruchtlose Gewissensbisse, erzeugen $^{30}$. Erforderlich ist vielmehr die - für alle Kollektivgüter, an denen große Kollektive partizipieren, nur mit Hilfe rechtlicher Regelungen mögliche - Institutionalisierung eines Ordnungsrahmens, der die sozialverträgliche Nutzung und gegebenenfalls erforderliche Pflege des jeweiligen Kollektivgutes ohne Überstrapazierung der moralischen Leistungsfähigkeit der Beteiligten ermöglicht ${ }^{31}$.

30 Diesen Satz habe ich mit Bedenken zu Papier gebracht, weil er als Kritik faktisch geltender Umwelt-Alltagsmoral, d.h. so mißverstanden werden könnte, als hielte ich diese für bereits überspannt. Was kritisiert werden soll, ist nicht eine Überspannung geltender Umweltmoral, von der keine Rede sein kann, sondern die Tendenz, ohne Rücksicht auf die Grenzen der Leistungsfähigkeit nicht institutionell vermittelter Umweltmoral dieser Steuerungsaufgaben zu überwälzen, die keine noch so angespannte Moral bewältigen kann. Über die Frage, ob die Umwelt-Alltagsmoral in Deutschland sich durch Überspannung auszeichnet oder nicht, herrscht freilich kein Konsens; s. etwa Wolfgang Pauser, „Margarine, Waschmaschine, Über-Ich“, DIE ZEIT vom 15. August 1997, S. 49. Herr Pauser sieht sich moralischer Überforderung unter anderem schon dadurch ausgesetzt, daß die Bezeichnung seiner Waschmaschine („Öko-Lavamat") ihn auf die Umweltrelevanz seiner Wäschepflege aufmerksam macht.

31 In diesem Sinne für den Bereich des Umweltschutzes Frey (Fn. 19), S. 47 f.; s. auch die übrigen in Fn. 19 Angeführten. Das Verhältnis von individueller Moral und Institutionen beschäftigt gegenwärtig weniger die Philosophie und die Soziologie, für die das Thema sich mit Luhmann im wesentlichen erledigt zu haben scheint, als vielmehr die Ökonomie und die ökonomisch basierte Wirtschaftsethik; s. statt vieler Karl Homann, Normativität angesichts systemischer Sozial- und Denkstrukturen, in: Wulf Gaertner (Hrsg.), Wirtschaftsethische Perspektiven IV, 1998 (im Erscheinen), m.w.N., sowie, an Arbeiten Homanns anschließend, vorzüglich Andreas Suchanek, Chancen und Grenzen der Implementation umweltverträglicher Verhaltensmuster in der modernen Gesellschaft, in: Matthias Sellmann (Hrsg.), Umweltethik und ihre gesellschaftliche Vermittlung, 1997, S. 45 ff. und Gerecke (Fn. 7); 
Damit ist eine Forderung an das Nutzerkollektiv formuliert, der in bezug auf die Individuen des Kollektivs die Forderung entspricht, sich als gute Bürger zu verhalten durch Mitwirkung am Aufbau und an der Erhaltung von Institutionen, die die erforderlichen Steuerungs- und Ordnungsleistungen erbringen ${ }^{32}$. In bezug auf den Umweltschutz sind dies unter anderem, ja sogar ganz wesentlich, die Institutionen eines wirksamen umweltschützenden Rechts. Die allgemeine Forderung, sich in bezug auf den Aufbau funktionsfähiger Institutionen als gute Bürger zu verhalten, ist freilich nicht sinnvoll in strikte und konkrete moralische Forderungen an die Adresse des einzelnen Bürgers übersetzbar. Keine Morallehre kann angeben, wer genau was genau zu tun hat, um zu vernünftigen institutionellen Lösungen beispielsweise für verkehrsbedingte Umweltprobleme zu kommen. Schon die Beantwortung der Frage, welche institutionellen Lösungen hier überhaupt vernünftig wären, fällt nicht primär ins ethische $\mathrm{Fach}^{33}$. Und selbst wenn als feststehend unterstellt wird, welche Rahmenbedingungen des Verkehrsverhaltens wie geändert werden sollten, sind aus der Einsicht, daß entsprechend gehandelt werden muß, keine konkreten individuellen Rollenzuweisungen und Aktivitätspflichten ableitbar. Nicht jeder kann und muß sich als Verkehrsexperte im Deutschen Bundestag für höhere Mineralölsteuern und Verbesserungen des Angebots der öffentlichen Verkehrsträger einsetzen, nicht jeder kann und muß verkehrsberuhigte

vgl. auch zahlreiche weitere Beiträge zum Thema in der Reihe „Wirtschaftsethische Perspektiven“ (Schriften des Vereins für Sozialpolitik) sowie Peter Ulrich, Integrative Wirtschaftsethik. Grundlagen einer lebensdienlichen Ökonomie, 1997.

32 In diesem Sinne, bezogen auf den Umweltschutz und die Rolle des Unternehmers, der ,bei noch nicht im Ordnungsrahmen angemessen reflektierten Umweltbeeinträchtigungen zum politischen Unternehmer werden" müsse, und mit Verweis auf die besondere Verpflichtung der Unternehmerverbände in diesem Zusammenhang auch Rat von Sachverständigen für Umweltfragen, Umweltgutachten 1994, Rz. 126; für den Gedanken einer die „Geschäftsethik“ ergänzenden ,republikanischen Unternehmensethik“ (als Ethik der unternehmerischen „Mitverantwortung“ für die ordnungspolitischen Rahmenbedingungen der eigenen Wirtschaftstätigkeit) Ulrich (Fn. 31), S. $427 \mathrm{ff}$.

33 Dasselbe gilt in vielen Fällen bereits für die Identifikation, und regelmäßig für die genaue Beschreibung, des jeweiligen Problems; näher dazu Hermann Lübbe, Moral und Moderne, in: ders., Modernisierung und Folgelasten, 1997, S. 131 (141 f.). 
Wohnquartiere planen, oder sich in einer Bürgerinitiative dafür einsetzen, ja nicht einmal jeder kann und muß sich ein fundiertes eigenes Urteil darüber bilden, ob schwach besetzte Nahverkehrszüge tatsächlich umweltfreundlicher sind als der Transport ihrer Insassen im motorisierten Individualverkehr.

Diese Diffusität des in bezug auf vernünftige Institutionenbildung von Individuen konkret Erwartbaren erschwert die Zuschreibung individueller Verantwortung für institutionell vermittelte oder institutionell nicht behobene Mißstände, jedenfalls wenn man den Verantwortungsbegriff im oben erläuterten, die Anlastung von Konsequenzen implizierenden Sinn gebrauchen will ${ }^{34}$. Daraus folgt nicht, daß auf dem Feld der Institutionenbildung für moralische Antriebe kein Bedarf oder keine Möglichkeit der Mobilisierung bestünde und Unterscheidungen zwischen achtungswürdigem, indifferentem und mißachtungswürdigem Individualverhalten sich nicht treffen oder nicht auf Regeln bringen ließen, kurz: daß moralische Kategorien hier prinzipiell fehl am Platze wären. Es besteht aber Anlaß zur Vorsicht beim Einsatz moralischer Verhaltenskritik. Dies gilt nicht nur im Hinblick auf die angesprochenen Probleme individueller Zurechnung. Richtiges Verhalten in bezug auf die Bildung und Erhaltung vernünftiger Institutionen hängt nicht nur von den moralischen Dispositionen der Akteure, sondern in besonders hohem Maße von ko-

$34 \mathrm{Zu}$ Problemen der Verantwortungszuschreibung in komplexen Zusammenhängen allg. Weyma Lübbe (Hrsg.), Kausalität und Zurechnung. Über Verantwortung in komplexen kulturellen Prozessen, 1994. Wenn demgegenüber Arbeiten zur Sozialethik auf der Existenz individueller Verantwortung für komplexe, institutionell vermittelte Verhältnisse beharren, wird dabei ein anderer als der oben explizierte und empfohlene Verantwortungsbegriff zugrundegelegt, nämlich der ebenfalls schon angesprochene extensivere, rein moralisch-appellative, wie ihn Hans Jonas verwendet, s. etwa Arthur Rich, Wirtschaftsethik, 1984, S. 56 ff., und, an Rich anknüpfend, Hans G. Nutzinger, Der Begriff Verantwortung aus ökonomischer und sozialethischer Sicht, in: Karl Homann (Hrsg.), Aktuelle Probleme der Wirtschaftsethik, 1992, S. 43 ff. Für die plausible Annahme, daß die Ausbreitung des (extensiven) Verantwortungsbegriffs historisch durch komplexitätsbedingte Schwierigkeiten des Umgangs mit traditionelleren Zurechnungskategorien wie Pflicht, Haftung, Schuld etc., d.h. gerade auch durch das im Text angesprochene Zurechnungsproblem mitveranlaßt ist, s. Franz-Xaver Kaufmann, Risiko, Verantwortung und gesellschaftliche Komplexität, in: Bayertz (Fn. 14), S. 72 ff. (85 ff.). 
gnitiven Voraussetzungen, von der - häufig kontroversen und oft auch prinzipiell unaufklärbaren - Richtigkeit zugrundegelegter Annahmen und Prognosen über gesellschaftliche und außergesellschaftliche Sachverhalte und deren Steuerbarkeit ab. Differenzen über institutionell richtiges Vorgehen müssen und können deshalb nicht ohne weiteres als moralische Differenzen gedeutet werden. Sachlicher Auseinandersetzung und damit der Auffindung institutionell vernünftiger Lösungen förderlicher ist es jedenfalls, wenn die Beteiligten auf der Basis der Prämisse operieren, daß bestehende Differenzen sachlicher Natur sind ${ }^{35}$.

Wenn hier in bezug auf den Umweltschutz und in bezug auf die Nutzung von Kollektivgütern im allgemeinen für eine Moral der Mitwirkung an vernünftigen institutionellen Lösungen plädiert wird, zielt das deshalb nicht etwa auf eine Moralisierung der Politik. Es wird keineswegs empfohlen, nun die politische Auseinandersetzung über vernünftige institutionelle Lösungen als Stätte des Kampfes zwischen gut und böse, oder doch wenigstens zwischen gut und weniger gut, zu interpretieren. Um Mißverständnisse in dieser Richtung zu vermeiden, sollte wohl besser von einer Kultur als von einer Moral der Mitwirkung an vernünttigen institutionellen Lösungen die Rede sein. Der mit diesem terminologischen Wechsel verbundene Perspektivenwechsel hat auch noch andere Vorteile. Die Thematisierung von Fragen guter Verhaltensordnung als Fragen der Moral führt, gleich von welchem Moralbegriff man ausgeht, stets auf definitorische Probleme, auf Probleme der Abgrenzung zu Verhaltensdeterminanten nicht moralischer Art, auf die es für die praktischen Zwecke der Institutionalisierung guter Verhaltensordnungen nicht ankommt. Unter welchen Voraussetzungen ein Verhalten wirklich als moralisches gelten kann, ob dazu „die Idee der Pflicht“" und damit Vernunft die alleinige Triebfeder sein $\mathrm{mu} \beta$, ob es auf vollkommene Uneigennützigkeit ankommt (und was dies alles eigentlich genau bedeutet), ob und wie Moral von Sitte, Mo-

35 Allgemeiner zum konfliktverschärfenden Potential moralischer Unterfütterung politischer Positionen und fưr eine daraus abgeleitete Skepsis gegen die Moralisierungstendenz, die von der philosophischen Umweltethik ausgeht, Paul B. Thompson, Pragmatism and Policy. The Case of Water, in: Light/Katz (Fn. 7), S. $187 \mathrm{ff}$. (205). 
de usw. abzugrenzen ist - alle diese mit jeder Verwendung des Moralbegriffs aufgeworfenen Fragen laufen in einem gewissen Sinne schon als Fragen dem Anliegen vernünftiger Institutionenbildung zuwider, weil sie auf analytische Trennung dessen zielen, was vernünftige Institutionen gerade zusammenführen sollen bzw. als zusammengehörig voraussetzen. Die Behandlung von Fragen der Institutionenbildung und des richtigen institutionenbezogenen Verhaltens als Fragen institutioneller Kultur statt als Fragen institutioneller Moral paßt insofern besser zur institutionenbezogenen Perspektive.

Die damit verbundene Entlastung von prekären Unterscheidungen hat Vorzüge auch auf der oben schon angesprochenen Ebene individueller Verhaltenskritik. Verhaltenskulturelle Defizite können zum Beispiel behauptet und begründet werden, ohne daß dies unbeweisbare und kommunikativ unzweckmäßige Hypothesen über einen entweder kognitiven oder moralischen Ursprung des Problems implizierte.

Dies ist nun der Ort, um auf die oben erwähnten Fallbeispiele aus Diskussionsveranstaltungen zurückzukommen. Worin liegt das in diesen Beispielsfällen zutage getretene Defizit an institutioneller Kultur? In beiden Fällen haben die Diskussionspartner, die sich über das ihrem Berufsstand in Gestalt institutioneller Regelungen oder Regelungsvorschläge entgegengebrachte "Mißtrauen“ ungehalten zeigten, individuellmoralische Lösungen in einem Zusammenhang offeriert, in dem nicht erwartet werden kann, daß solche Lösungen funktionieren. Von konkurrierenden Wirtschaftsunternehmen kann nicht erwartet werden, daß sie die Einhaltung geltender umweltrechtlicher Regelungen unter hohen Kosten gewissenhaft selbst überwachen und gewährleisten, wenn und soweit weder ihnen selbst noch der Konkurrenz behördliche Überwachung und Sanktionierung festgestellter Verstöße oder irgendwelche anderen unliebsamen Konsequenzen drohen. Die unter solchen Umständen sinnvolle institutionelle Lösung, nämlich eine behördliche Überwachung, die unter anderem die Funktion hätte, Normkonformitätsbereitschaft zu erzeugen durch Stärkung des Vertrauens, daß auch die Konkurrenz sich an die geltenden Regeln halten wird - dieser vernünftigen institutionellen Lösung haben beide Diskussionspartner unter Verweis auf angeblich ausreichende moralische Kapazitäten ihre Zustimmung verweigert. Im 
Ergebnis führt die mit dieser Argumentation nahegelegte Regulierungsstrategie bzw. Strategie der Nichtregulierung geradewegs zur Erosion derjenigen Moralbestände, auf die dabei vertraut wird.

5. Möglichst viel moralische Selbststeuerung, möglichst wenig rechtliche Fremdsteuerung?

Wenn nun individuelle Moral für sich genommen, ohne rechtliche Flankierung, in bezug auf den Schutz der Umwelt wenig ausrichten kann, rechtliche Lösungen und die dazugehörigen spezifischen Instrumente der Rechtsdurchsetzung also unentbehrlich sind - gibt es aber nicht Gründe, rein moralisch motiviertem Verhalten eine intrinsische Vorzugswürdigkeit gegenüber rechtsgesteuertem Verhalten, oder moralischer Motivation eine intrinsische Vorzugswürdigkeit gegenüber rechtlicher Steuerung, zuzuschreiben, die zum Verzicht auf rechtliche Lösungen auch dann veranlassen sollte, wo diese in bezug auf den Umweltschutz wirksamer sind als rein moralische Antriebe?

\section{a) Recht als Amoralisierungsfaktor?}

\section{aa) Bedenken}

Zunächst einmal könnte man sich fragen, ob nicht ein gewichtiger Nachteil jeder rechtlichen Steuerung darin liegt, daß sie etwa vorhandene intrinsische Motivationen zu richtigem Handeln durch extrinsische, von außen kommende Anreize überformt - eben Moral durch Recht ersetzt. Sollte nicht, mit anderen Worten, der Einsatz rechtlicher Steuerungsinstrumente immer schon allein deswegen möglichst vermieden werden, weil damit immer Gelegenheiten zu moralischer Bewährung vernichtet werden?

Dieses Bedenken ist in der Vergangenheit oft gegen die Schaffung sozialer Institutionen, insbesondere gegen die Schaffung sozialstaatlicher Institutionen geltend gemacht worden. Die dahinter aufscheinen- 
de Auffassung unseres irdischen Daseins als einer Bewährungsprobe, die nicht erleichtert, sondern bestanden werden soll, ist den meisten von uns aber heute fremd und wird vor allem den Jüngeren so fremd sein, daß ich hier darauf verzichten kann, gegen sie zu argumentieren. Die Abneigung gegen das den Bedarf an caritas, an wohltätiger Nächstenliebe, reduzierende Potential rechtlich statuierter institutioneller Problemlösungen hatte ihre Wurzeln vor allem in der älteren katholischen Tradition ${ }^{36}$. Dem Protestantismus ist diese Betrachtungsweise dagegen eher fremd ${ }^{37}$. Systematisch entwickelt finden wir den moderneren, protestantischen Standpunkt bei Hegel, dessen ganze praktische Philosophie als eine Auseinandersetzung mit katholischen Heiligkeitsidealen, katholischer Opfermoral, verstanden werden kann. Hegel setzt diesen das Ideal der Sittlichkeit entgegen, das nur in vernünftigen Institutionen zu verwirklichen ist - Institutionen nämlich, deren vernünftiger, sittlicher Charakter gerade darin besteht, daß in ihnen und in ihrem Zusammenspiel das Allgemeininteresse und die Interessen der je besonderen Individuen gleichermaßen befriedigt werden, also gerade nicht das eine dem anderen aufgeopfert werden $\mathrm{mu} \beta^{38}$.

Mit einer ganz anderen als der eben besprochenen weltanschaulichen Begründung finden wir Bedenken, die sich auf die Verdrängung intrinsi-

36 Eine Wende ist hier mit den großen päpstlichen Sozialenzykliken, vor allem mit der Enzyklika Quadragesimo anno (1931), vollzogen, die die Notwendigkeit rechtlicher Institutionen zur Verbesserung der Lage der Arbeiter anerkennt und Auffassungen, die hier ausschließlich ein Betätigungsfeld für Werke freiwilliger christlicher Nächstenliebe sehen wollen, ausdrücklich zurückweist. S. dazu die Erläuterungen bei Oswald von Nell-Breuning, Die soziale Enzyklika, 3. Aufl. 1950, S. $141 \mathrm{ff}, 231 \mathrm{ff}$.

37 Problematisierungen des modernen Sozialstaates unter dem Gesichtspunkt, daß er „die Nächstenliebe ... überflüssig zu machen“, eine „Institutionalisierung der Mitmenschlichkeit" $\mathrm{zu}$ bewirken drohe, findet man allerdings gelegentlich auch in der lutherischen Sozialethik, s. die Wiedergabe bei Hans Berthold, Sozialethische Probleme des Wohlfahrtstaates, 1968, S. 11 ff. (11, 23 u. passim; krit. dazu ders., aaO. S. 114 ff., m.w.N.); vgl. als Kontrapunkt Berthold Brecht, Flüchtlingsgespräche, Werkausgabe Bd. XIV der Ausgabe Frankfurt a.M. 1967, S. 1472: „Sie sind sicherer in einem Lande, wo's keine Nächstenliebe braucht, damit sie kuriert werden“".

38 Näher dazu Gertrude Lübbe-Wolff, Die Sittlichkeit in der bürgerlichen Gesellschaft. Hegels Wegweisung durch das Nadelöhr, in: Archiv für Rechts- und Sozialphilosophie, Bd. 68, 1982, S. $223 \mathrm{ff}$. 
scher durch extrinsische Motivation und, damit zusammenhängend, von Moral durch Recht, beziehen, in der ökonomischen Literatur formuliert. Vor allem der Zürcher Ökonom Bruno S. Frey und eine Reihe von CoAutoren haben diesbezügliche Überlegungen speziell mit Bezug auf die Umweltpolitik angestellt ${ }^{39}$. Frey \& Co. sehen das Problem nicht darin, daß Recht Gelegenheiten zu moralischer Bewährung vernichtet. Sie schreiben intrinsischer, moralischer Motivation keine prinzipielle Vorzugswürdigkeit gegenüber extrinsischer, durch Recht vermittelter, $\mathrm{zu}^{40}$. Sie betrachten die Frage, ob man sich auf Moral verlassen oder rechtliche Instrumente einsetzen sollte, einfach unter dem Gesichtspunkt der Steuerungseffizienz und kommen zu dem Ergebnis, daß der Einsatz rechtlicher Instrumente infolge der damit verbundenen Verdrängung intrinsischer (moralischer) durch extrinsische (rechtlich induzierte) Motivation kontraproduktiv wirken $\mathrm{kann}^{41}$. Die Verdrängung ist aus der nüchternen ökonomischen Sicht, von der die Autoren ausgehen, kein Problem, solange und soweit eben dann anstelle der möglicherweise verdrängten moralischen Motivation der rechtlich gesetzte äußere Anreiz

39 Bruno S. Frey/Isabelle Busenhart, Umweltpolitik: Moral oder Ökonomie? in: Andreas Diekmann/Axel Franzen (Hrsg.), Kooperatives Umwelthandeln, 1995, S. 9 ff.; Bruno $S$. Frey/Felix Oberholzer-Gee, Zum Konflikt zwischen intrinsischer Motivation und umweltpolitischer Instrumentenwahl, in: Horst Siebert (Hrsg.), Elemente einer rationalen Umweltpolitik, 1996, S. 207 ff.; Bruno S. Frey/Friedrich Schneider, Wanum wird die Umweltökonomie kaum angewendet?, ZfU 1997, S. 153 ff., jew. m.w.N. Zur zugrundeliegenden psychologischen Theorie intrinsischer Motivation s. insbesondere Edward L. Deci, Intrinsic Motivation, 1975. Dem Kollegen Wulf-Uwe Meyer verdanke ich in diesem Zusammenhang u.a. den Hinweis, daß in der psychologischen Motivationsforschung ganz unterschiedliche Konzeptionen von intrinsischer Motivation entwickelt worden sind und verwendet werden; s. für einen Überblick über verschiedene Definitions- und Erklärungsansätze Heinz Heckhausen, Motivation und Handeln, 2. Aufl. 1989, S. $455 \mathrm{ff}$.

40 S. Frey/Oberholzer-Gee(Fn. 39), S. 228 Fn. 14.

41 Zum Problem der Zerstörung intrinsischer durch extrinsische Motivation auch Margit Osterloh, Vom Nirwana-Ansatz zum überlappenden Konsens: Konzepte der Unternehmensethik im Vergleich, in: Hans G. Nutzinger (Hrsg.), Wirtschaftsethische Perspektiven III, 1996, S. 203 ff.; Hannelore Weck-Hannemann, Rationale Außensteuerung menschlichen Umweltverhaltens - Möglichkeiten und Grenzen, in: Erik Gawel/Gertude Lübbe-Wolff (Hrsg.), Rationale Umweltpolitik - rationales Umweltrecht (erscheint demnächst). 
wirkt. Das Problem wird darin gesehen, daß der durch extrinsische Anreize ausgelöste moralverdrängende Effekt größer sein kann als der Steuerungseffekt, der von dem zugeführten extrinsischen Anreiz ausgeht, so daß sich insgesamt eine negative Bilanz ergibt.

Um die Relevanz dieses Problems für die Umweltpolitik richtig einschätzen zu können, muß man sich Klarheit über die Ursachen einer möglichen Verdrängung moralischer Motivation durch extrinsische Anreize verschaffen.

Freys Hypothese dazu lautet, daß der Abbau moralischer Motivation bei hinzutretenden äußeren Anreizen als Reaktion auf die entstandene "Überveranlassung" zu deuten sei: der Betroffene habe ,zu viele“ Motive und reduziere deshalb die in seiner Kontrolle liegende intrinsische Motivation. Deshalb werde etwa der Sohn einer Familie, der zunächst, intrinsisch motiviert, aus eigener Initiative den Rasen gemäht habe, seine intrinsische Motivation abbauen, wenn man anfange, ihn für das Rasenmähen zu bezahlen, und dann später auch nicht mehr aus eigenem Antrieb mähen, wenn die Bezahlung wieder ausbleibe ${ }^{42} . \mathrm{Ge}$ gen die hier offenbar zugrundeliegende Vorstellung, daß Motivation zu einer Art Belastung wird, für die ein Abbaumechanismus einsetzt, sobald das zur Veranlassung einer bestimmten Handlungsweise notwendige Maß überschritten ist, spricht aber schon die darin implizierte Verkehrung des Verhältnisses von Motivation und Handlung. Plausibler scheint es, relative Unterveranlassung, Erwartungsentlastung und Frustration als erklärende Faktoren in Betracht zu ziehen ${ }^{42 a}$.

Im Falle des öfter beobachteten Abfalls moralischer Leistungsfähigkeit nach dem Entzug von vorübergehend gewährten Belohnungsanreizen, im Fall des jetzt nicht mehr so gern den Rasen mähenden Sohnes also beispielsweise, dürfte eher die entzugsbedingte relative Unterveranlassung als eine zwischenzeitlich als ,Zuviel" verspürte und daher abgebaute „Überveranlassung“ das Problem sein. Wer mit einer Arbeit

42 Frey/Busenhart (Fn. 39), S. 16.

42a Die nachfolgenden Hypothesen sind nicht deckungsgleich, soweit ersichtlich aber auch nicht inkompatibel mit den Ergebnissen der einschlägigen motivationspsychologischen Forschung; vgl. Bernard Weiner, Motivationspsychologie, 3. Aufl. 1994, S. 203 ff. 
zwanzig Mark pro Stunde verdient hat, wird sich für zehn Mark vielleicht nicht mehr in Bewegung setzen, während ein immer nur mit zehn Mark Entlohnter mit größerer Wahrscheinlichkeit durch diese Summe auch weiterhin ausreichend motiviert sein wird. Ähnlich wie dem von zwanzig auf zehn Mark gesetzten Lohnempfänger wird es dem rasenmähenden Sohn gehen, wenn man ihm einen Teil seiner Antriebsfaktoren, nämlich den finanziellen, nachträglich wieder entzieht. Diese Abhängigkeit der Motivationslage vom jeweils Gewohnten soll im folgenden nicht weiter behandelt werden, weil damit zusammenhängende Phänomene der Moral,,verdrängung" durch extrinsische Anreize immer erst zum Problem werden, wenn der extrinsische Anreiz später wieder entfällt. Ein für umweltpolitische Regulierung relevantes Problem besteht hier also, da umweltpolitische Regulierung auf Konstanz angelegt ist, nicht.

Auch die Erwartungsentlastung, die mit dem Einsatz extrinsischer Anreize, und zwar insbesondere mit dem Einsatz positiver materieller Anreize wie z.B. Geldzahlungen, verbunden sein kann, dürfte im Zusammenhang mit dem Einsatz allgemeiner, auf umweltgerechtes Verhalten zielender Regulierungsinstrumente kaum relevant werden. Je nach Situationskontext kann es Gründe geben, die Bereitstellung extrinsischer Anreize als Indiz dafür zu interpretieren, daß moralische Ansprüche nicht gestellt, moralisch motivierte Verhaltensweisen also gar nicht erwartet werden. Dies erklärt vielleicht am ehesten den von Frey als Beispiel für Moralverdrängung angeführten Fall einer Schweizer Gemeinde, in der zunächst vergleichsweise hohe Bereitschaft bestand, die Plazierung eines Atommüll-Endlagers im Gemeindegebiet zu akzeptieren, dann aber die Zustimmung drastisch absank, als die Behörden Kompensationszahlungen anboten ${ }^{43}$. Die Gemeindebürger werden dieses Angebot, wenn nicht sogar als Indiz für die Existenz entschädigungsbedürftiger Risiken, die sie zuvor so nicht eingeschätzt hatten, dann doch jedenfalls als Indiz dafür verstanden haben, daß man nicht Bürgersinn, sondern Geschäftssinn von ihnen erwartete, und als Geschäft war ihnen die Sache, einmal so umdefiniert, dann of-

Frey/Schneider (Fn. 39), S. 159, m.w.N. 
fenbar nicht attraktiv genug. Geschäfte dürfen, anders als Moral, ja auch anderen überlassen werden. Für die Befürchtung, daß irgendwelche praktisch überhaupt in Frage kommenden extrinsischen Anreize zu umweltgerechtem Verhalten in Gestalt allgemeiner umweltpolitischer Regulierungen auf diese Weise, per Erwartungsentlastung, in einem problematischen Ausmaß moralverdrängend wirken könnten, gibt es aber wenig Anlaß, denn regulatorische Anreize zu umweltgerechtem Verhalten haben objektiv nicht moralerübrigende, sondern moralunterstützende Funktion, und dies dürfte auch den jeweiligen Adressaten im Regelfall evident, zumindest aber ohne weiteres zu vermitteln sein (s. dazu noch unter bb).

Zur dritten Ursache möglicher Moralverdrängung: Steuerung über extrinsische Anreize, und zwar vor allem die Steuerung über negative extrinsische Anreize wie sanktionierte Ge- und Verbote und darauf bezogene Kontrollen, kann moralische Leistungsbereitschaft reduzieren, indem sie frustrierend wirkt. Frustrationsbedingte moralische Demotivation ist aber keine automatische Folge jeder Fremdregulierung und Fremdkontrolle, sondern tritt da und nur da auf, wo die Regulierung und Kontrolle als Verweigerung angemessenen Vertrauens oder angemessener Wertschätzung erbrachter moralischer Leistungen interpretiert wird. Dies erklärt die Beobachtung, daß moralische Demotivation durch Fremdsteuerung und Fremdkontrolle vor allem im sozialen Nahbereich, weniger dagegen in anonymisierten und unpersönlichen Beziehungen eine Rolle spielt ${ }^{44}$. So wird beispielsweise seine Kinder nicht zur Ehrlichkeit, sondern im Gegenteil zur Unehrlichkeit motivieren, wer ihnen durch ständiges In-Zweifel-Ziehen und Kontrollieren zu verstehen gibt, $\mathrm{da} ß$ er ihnen die Neigung unterstellt, zu lügen und zu betrügen. Niemand wird sich aber andererseits dadurch moralisch demotiviert fühlen, daß er in der Bahn dem Schaffner eine Fahrkarte vorweisen muß, obwohl natürlich auch dies ein Zeichen eingeschränkten Vertrauens der Bahn zu ihren Fahrgästen ist. Man versteht eben, weshalb Fahrkartenkontrollen für die Funktionsfähigkeit der Bahn erforderlich sind, und interpretiert sie deshalb nicht als Verweigerung angemessenen Vertrauens.

44 Frey/Oberholzer-Gee (Fn. 39), S. 222., m.w.N. 
Konstellationen, in denen umweltrechtliche Regelungen und ein sachgerechtes Ausmaß an darauf bezogener Kontrolle in nachvollziehbarer Weise als Verweigerung angemessenen Vertrauens aufgefaßt werden und deshalb mehr moralischen Schaden als rechtlichen Nutzen stiften könnten, sind schwer vorstellbar. Nehmen wir ein von Frey und Oberholzer-Gee verwendetes Beispiel: „Viele private Haushalte haben z.B. schon seit langem ihren Abfall sorgfältig getrennt und umweltfreundlich entsorgt. Wenn nun der Staat allen Haushalten eine ganz bestimmte Art der Trennung und Entsorgung des Hausmülls zwingend vorschreibt, wird den bisher umweltmoralisch motivierten Haushalten die Möglichkeit genommen, ihrer intrinsischen Motivation nachzuleben. Diese wird überflüssig und durch äußeren Zwang substituiert ${ }^{445}$. Frey und Oberholzer-Gee qualifizieren dies als eine ,schädliche Wirkung auf die Umweltmoral“".

Folgt man allerdings ihrer eigenen Bekundung, nach der sie intrinsische Motivation nicht für per se besser halten als extrinsische, dann läge in der Tatsache, daß in etlichen Haushalten, die bisher aufgrund moralischer Motivation ihren Müll sortiert haben, der Müll jetzt auch oder vielleicht sogar nur noch aufgrund externer rechtlicher Anreize getrennt wird, an sich kein Schaden. Solange die rechtliche Regelung im Ergebnis dazu führt, daß mehr Haushalte als zuvor ihren Müll trennen, im Ganzen also eine Verbesserung in Richtung auf die verfolgte abfallwirtschaftliche Zielsetzung eintritt, spricht unter dieser Prämisse die Tatsache, daß in dem einen oder anderen Haushalt die rechtliche Regelung intrinsische Sortiermotivation verdrängt haben mag, nicht gegen diese rechtliche Regulierung. Als ein Problem dürften die Autoren - immer unter der gewählten Prämisse, daß moralischer Motivation keine intrinsische Vorzugswürdigkeit zugeschrieben wird - einen eventuellen Verdrängungseffekt erst dann qualifizieren, wenn Demotivation in einem den Steuerungseffekt der rechtlichen Regelung insgesamt konterkarierenden Ausmaß ausgelöst würde, wenn also beispielsweise zuvor schon mehr als $50 \%$ der Haushalte ihren Abfall freiwillig sortiert hätten und nun die ganze Heerschar der frustrierten

45 Frey/Oberholkzer-Gee (Fn. 39), S. 227. 
freiwilligen Abfallsortierer sich entschlösse, die neu eingeführte Rechtspflicht zu boykottieren, oder wenn der Ärger über Mißachtung des an den Tag gelegten freiwilligen Umweltengagements die Motivation zu umweltfreundlichem Handeln nun auch auf anderen und noch wichtigeren Gebieten als dem der Abfallsortierung lahmlegte oder gar in ihr Gegenteil verkehrte ${ }^{46}$. Diese Szenarien sind aber so wirklichkeitsfern, daß man sich geniert, sie überhaupt zur Sprache zu bringen.

\section{bb) Moral ermöglichende und Moral unterstützende Funktionen des Rechts}

Das von Frey und Oberholzer-Gee benutzte Beispiel scheint mir eher geeignet, das genaue Gegenteil des behaupteten Verdrängungseffekts $\mathrm{zu}$ illustrieren. Es handelt sich nämlich um ein gutes Beispiel dafür, daß Moral durch Recht überhaupt erst ermöglicht wird, genauer: daß viele Möglichkeiten sinnvollen umweltmoralischen Verhaltens von Voraussetzungen abhängen, die nur mit Hilfe von Recht überhaupt geschaffen werden können.

Abfallsortierung beispielsweise hat, abgesehen von den vergleichsweise wenigen Haushalten, die über einen eigenen Garten verfügen und daher zumindest ihre kompostierbaren Abfälle sinnvoll selbst verwerten können, nur einen Sinn, wenn die sortierten Abfälle den Haushalten auch als sortierte abgenommen und getrennten Verwertungen bzw. Beseitigungswegen zugeführt werden ${ }^{47}$. Diese differenzierte Abnahme und Entsorgung der verschiedenen Abfallfraktionen ergibt sich, weil sie eo ipso keinen Gewinn abwirf, nicht von selbst, sondern muß auf der Grundlage von Rechtsvorschriften organisiert werden; sie wäre übrigens auch faktisch völlig ausgeschlossen, solange jeder Haushalt sei-

46 Diese zuletzt genannte Variante (über den Eingriffsbereich hinauswirkende Verdrängung durch externe Eingriffe) bezeichnen Frey und Oberholzer-Gee als „motivationalenÜberschwappeffekt", aaO. (Fn. 39), S. 228 ff.

47 Dazu und allgemeiner für die Notwendigkeit organisierter Bereitstellung von Verhaltensangeboten für die Umsetzung vorhandener umweltmoralischer Motivation in konkretes Handeln Meinolf Dierkes/Hans-Joachim Fietkau, Umweltbewußtsein Umweltverhalten, 1988, S. $163 \mathrm{ff}$. 
ne Sortieranweisungen aus der Umweltethik schöpfte. Ein System differenzierter Erfassung, Verwertung und Beseitigung unterschiedlicher Haushaltsabfallfraktionen mit der ganzen dazugehörigen aufwendigen Infrastruktur für die Bereitstellung, Abfuhr und Behandlung der Abfälle in Anlagen, für die die Investitionskosten in die Hunderte Millionen gehen, kann aber gar nicht auf die Beine gestellt werden, wenn es allein der Umweltmoral der Abfallerzeuger überlassen bleibt, ob und wie sie sich mit eigenen Sortierleistungen an dem System beteiligen wollen. Ohne allgemeine, rechtliche Vorgaben für die Abfallsortierung sind also die Voraussetzungen dafür, daß entsprechende moralische Antriebe sinnvoll zur Geltung kommen können, gar nicht herstellbar.

Diese Funktion des Rechts, umweltmoralische Verhaltensmöglichkeiten überhaupt erst zu eröffnen, scheint mir sehr viel hervorhebenswerter als der von Frey und Oberholzer-Gee problematisierte motivationspsychologische Verdrängungseffekt. Das umweltbezogene Recht ist voll von Bestimmungen, die in ähnlicher Weise den Raum der Möglichkeiten umweltmoralischen Verhaltens nicht etwa verengen, sondern im Gegenteil erweitern.

Rechtlich geordnete Produktkennzeichnungen sind z.B. in vielen $\mathrm{Be}-$ reichen Voraussetzung dafür, daß eine vorhandene Bereitschaft der Konsumenten, Umweltgesichtspunkte beim Einkauf zu berücksichtigen, auch tatsächlich zur Geltung gebracht werden kann. Viele Möglichkeiten des bürgerschaftlichen Engagements in Umweltfragen hängen ebenfalls davon ab, daß das Recht entsprechende Möglichkeiten eröffnet. Ich erwähne nur den gesetzlichen Anspruch auf Zugang zu behördlichen Informationen über die Umwelt, die - in Deutschland nur sehr eingeschränkt vorhandenen - Möglichkeiten der Verbandsklage, mit der Umweltverbände sich für die Einhaltung geltender Umweltschutzvorschriften einsetzen können, und die durch das geltende Umweltrecht eröffneten Möglichkeiten der Öffentlichkeitsbeteiligung in umweltrelevanten Planungs- und Genehmigungsverfahren, die nicht nur für die Verfolgung privater Interessen, sondern auch für das von solchen Interessen ganz unabhängige Geltendmachen von Umweltschutzgesichtspunkten im jeweiligen Verfahren genutzt werden können. 
Auch abgesehen von solchen Rechtsvorschriften, durch die bestimmte Möglichkeiten umweltmoralischen Verhaltens überhaupt erst geschaffen werden, setzt die Charakterisierung des Verhältnisses von Recht und Moral als Verdrängungsverhältnis die Sache nicht in das richtige Licht. Wo ein Verhalten, das einige bereits aus moralischer Überzeugung an den Tag gelegt haben, zusätzlich auch noch rechtlich vorgeschrieben wird, macht dies die entsprechenden moralischen Verhaltensantriebe ja keineswegs überflüssig. Dazu sind die extrinsischen Verhaltensanreize, die das Rechtssystem bereitstellen kann, in aller Regel zu schwach ${ }^{48}$.

Von einer rechtlichen Geschwindigkeitsbegrenzung für den Autoverkehr beispielsweise geht kein faktischer Zwang zur Einhaltung der Geschwindigkeitsbegrenzung aus. Fahrer können sich Informationen über die Standorte von Überwachungskameras verschaffen oder auf andere Weise nicht kontrollierte Strecken zu identifizieren versuchen, um ungehindert und unsanktioniert möglichst schnell fahren zu können, und sie können auch bereit sein, für ungebremstes Fahrvergnügen hier und da ein Bußgeld zu investieren. Wer bislang schon freiwillig aus ökologischen Gründen langsamer gefahren war, dessen moralische Motivation wird also durch die Einführung einer Rechtspflicht und das damit verbundene Sanktionsrisiko keineswegs überflüssig, sondern nur weniger strapaziert: $\mathrm{zu}$ dem bisher schon vorhandenen intrinsischen Motiv tritt ein weiteres hinzu, und die moralische Anstrengung des Langsamfahrens, die nicht zuletzt darin bestanden hatte, daß man sich dauernd von moralisch weniger anstrengungswilligen Mitmenschen hatte überholen lassen müssen, wird durch die rechtliche Regelung reduziert, weil und sofern sie das Prinzip des Langsamfahrens (auch faktisch) verallgemeinert. Und für viele Andere, die sich bislang zum langsameren Fahren noch nicht entschließen konnten, obwohl ihnen der gute Sinn langsameren Fahrens deutlich war, beseitigt erst diese rechtliche Verallgemeinerung das Hindernis, das ihrer moralischen Motivation zu maßvollerer Geschwindigkeit bislang im Wege gestan-

48 Dies wird auch in der "Verdrängungsdiskussion“ gesehen, vgl. Frey/Schneider (Fn. 39), S. 158, m.w.N. 
den hatte, nämlich die Freiheit aller anderen, schneller zu fahren ${ }^{49}$. Diese Zusammenhänge legen es sehr viel näher, von einer Moral unterstützenden als von einer Moral verdrängenden Funktion des Rechts $\mathrm{zu}$ sprechen $^{50}$.

\section{cc) Moralbedarf für die Konstitution vernünftigen Rechts}

Aversionen, die sich von der Annahme nähren, daß rechtliche Regulierung gleichbedeutend wenn nicht mit Demoralisierung, so doch wenigstens mit Amoralisierung sei, würden schließlich auch die Bedeutung von Moral für die Konstitution vernünftigen Rechts ${ }^{51}$ vernachlässigen. Jede vernünftige rechtliche Regelung, im Umweltschutz wie auch sonst, kann nur auf der Grundlage zahlloser Institutionen der Wissensund Willensbildung zustandekommen, deren Funktions- und Leistungsfähigkeit auch auf moralischem Verhalten der Beteiligten beruht.

49 Vgl. die empirischen Befunde bei Horst W. Opaschowski, Freizeit und Umwelt, Hamburg (B.A.T. Freizeit Forschungsinstitut) 1985, (hier zitiert nach NunnerWinkler [Fn. 19], S. 142). Bürger sind danach zu umweltfreundlichem Verhalten ohne weiteres bereit, wenn es ihnen geringe Kosten verursacht. $\mathrm{Zu}$ größeren Verzichtsleistungen, beispielsweise zu Einschränkungen hinsichtlich des Autofahrens, sind sie dagegen nicht ohne weiteres, sondern nur unter der Voraussetzung bereit, $\mathrm{da} ß$ ein entsprechender Beitrag auch anderen abverlangt wird. Allgemeiner zu der Annahme, daß umweltmoralisches Verhalten im allgemeinen nur im Niedrigkostenbereich an den Tag gelegt wird (Low-Cost-Hypothese) Peter Preisendörfer/Axel Franzen, Der schöne Schein des Umweltbewußtseins, in: Andreas Diekmann/Carlo C. Jaeger, Umweltsoziologie, 1996, S. 219 ff. (234 f.); Frey/Schneider (Fn. 39), S. 156 f., jew. m.w.N.

50 Vgl. auch Nunner-Winkler (Fn. 19), S. 141, die in diesem Zusammenhang von der vertrauensbildenden Funktion des Rechts und der dazugehörigen Sanktionen spricht.

51 Diesen Gesichtspunkt hervorhebend Nunner-Winkler (Fn. 19), S. 126 f.; Erwähnung auch bei Frey/Schneider (Fn. 39), S. 158; s. außerdem zur Relevanz von Moral auch für die sachgerechte Anwendung rechtlicher Normen Lutz Eckensberger/Heiko Breit, Recht und Moral im Kontext von Kohlbergs Theorie der Entwicklung moralischer Urteile und ihrer handlungstheoretischen Rekonstruktion, in: Ernst-Joachim Lampe (Hrsg.), Zur Entwicklung von Rechtsbewußtsein, 1997, S. $253 \mathrm{ff}$. (258). 
Nicht zuletzt hängt das Zustandekommen vernünftiger Regelungen für den Umweltschutz davon ab, daß die Bürger als Wähler bereit sind, solche Regelungen, oder doch jedenfalls die Regierungsmacht derer, die sie erlassen, zu akzeptieren, auch wenn damit individuelle oder kollektive Lasten verbunden sind. Auch in diesem Akzeptieren stecken moralische Leistungen, die nicht übersehen und verachtet werden dürfen. Sie werden aber, wie alle die moralischen Leistungen, von denen die Funktionsfähigkeit komplexerer institutioneller Zusammenhänge abhängt, leicht verachtet oder übersehen, wenn man sich den Blick durch eine Theorietradition verengen läßt, die - im 18. Jahrhundert aus nachvollziehbaren Gründen - Moral- und Rechtslehre in strenger Abgrenzung voneinander behandelt und deshalb für Fragen der Moral des Verhaltens in rechtlich verfaßten Institutionen, wie überhaupt für viele Fragen des Zusammenhangs von Recht und Moral, wenig Interesse und Verständnis entwickelt hat.

\section{b) Recht, Moral und Freiheit}

Wie steht es aber mit dem Gesichtspunkt der Wahrung individueller Freiheit als einem Argument, das dafür sprechen könnte, bei der Verfolgung von Umweltschutzzielen eher auf Verbesserungen der allgemeinen Umweltmoral als auf rechtliche Regulierungen zu setzen ${ }^{52}$ ?

Der Gedanke, daß die Reichweite, das Gegenstandsfeld des Rechts von dem der Moral nach Gesichtspunkten der Freiheitssicherung abzugrenzen ist, hat Tradition. Kant weist dem Recht diejenigen und nur diejenigen Normen $\mathrm{zu}$, die der Sicherung gleicher äußerer Freiheit dienen. Nur die hierauf bezüglichen Pflichten sind Rechtspflichten, und nur für diese, nicht dagegen für bloße Tugendpflichten, ist ,eine äußere Gesetzgebung möglich“. Die bloßen Tugendpflichten ,können ... keiner äußeren Gesetzgebung unterworfen werden“"53.

Ich fürchte, daß es wenig Sinn hat, hier anzuschließen, zu prüfen, ob und inwieweit umweltbezogene Pflichten freiheitssichernden Charak-

52 Zu dieser Frage auch Kirsch (Fn. 19).

53 Kant (Fn. 16), A 47. 
ter haben, und nach Maßgabe des Ergebnisses dieser Prüfung für oder gegen eine Verrechtlichung zu plädieren. Es fehlt hier der Raum, um zu begründen, weshalb der Gesichtspunkt der Freiheitsgewährleistung, bei aller Bedeutung, die ihm zukommt, nicht und heute sehr viel weniger als zu Kants Zeiten sinnvoll zum zentralen und ausschließlichen Gesichtspunkt für die Bestimmung der möglichen Inhalte und damit auch der möglichen Reichweite von Recht gemacht werden kann ${ }^{54}$. Die Probleme eines wirksamen Umweltschutzes sind außerdem zu komplex, die denkbaren Lösungen zu planungs- und koordinationsbedürftig, als daß die Problemlösung vernünftigerweise ihren Ausgang von der Bestimmung und Qualifizierung individueller Pflichten nehmen könnte.

Nähern wir uns der Sache also von den Umweltproblemen her, die gelöst werden müssen. Angenommen - solche Annahmen sind natürlich ihrerseits höchst voraussetzungsvoll - es sei erforderlich, in Deutschland innerhalb eines gegebenen Zeitraums den $\mathrm{CO}_{2}$-Ausstoß um $35 \%$ zu senken ${ }^{55}$. Unter dieser Prämisse kann es offenbar nicht mehr um die Freiheit der Bürger und Bewohner Deutschlands gehen, ihren Energieverbrauch in der fraglichen Zeitspanne nicht um $35 \% \mathrm{zu}$ senken. Wenn aber diese Freiheit in jedem Fall beschränkt werden muß, dann stellt sich die Frage, wie die als notwendig erkannte Beschränkung dieser Freiheit erstens möglichst wirksam, zweitens auf eine ihrerseits möglichst freiheitliche und, drittens, auch sonstigen Ziel-

54 S. im übrigen für die schon von Hegel u.a. auch gegen Kant zur Geltung gebrachte Einsicht in die freiheitschaffende, den Raum der Möglichkeiten erweiternde Potenz des Rechts Gertrude Lübbe-Wolff, Die Grundrechte als Eingriffsabwehrrechte, 1988, S. $82 \mathrm{ff}$. Diese alte Erkenntnis ist in jüngerer Zeit reformuliert und auch auf außerrechtliche (moralische) Normen übertragen worden durch die ökonomische Theorie des Rechts und der Moral, s. für das Recht James M. Buchanan, The Limits of Liberty, 1974, S. $107 \mathrm{ff}$; für moralische Normen ders., The Economics and the Ethics of Constitutional Order, 1991, S. 179 ff.; David Gauthier, Morals by Agreement, 1986; s. auch Karl Homann, Ethik und Ökonomik, in: ders. (Hrsg.), Aktuelle Probleme der Wirtschaftsethik, 1992, S. 7 ff. (9).

$55 \mathrm{Vgl}$. die vom Wuppertal Institut für Klima, Umwelt und Energie erstellte Studie BUND/Misereor (Hrsg.), Zukunftsfähiges Deutschland, 1997, S. 56 ff., die die erforderliche Reduktion des $\mathrm{CO}_{2}$-Ausstoßes mit $35 \%$ bis 2010 und $80-90 \%$ bis 2050 berechnet hat. 
vorstellungen wie z.B. Minimierung von Wohlstandsverlusten, Gerechtigkeit der-Lastenverteilung etc. möglichst weitgehend entsprechende Weise bewerkstelligt werden kann.

Die verbreitete Vorstellung, daß Verhaltenssteuerung durch Recht dabei unter dem zweitgenannten Gesichtspunkt, unter dem Gesichtspunkt der Freiheitlichkeit also, prinzipiell schlecht abschneidet und deshalb möglichst vermieden werden sollte, gründet sich auf eine veraltete Vorstellung von der Wirkungsweise des Rechts. Diese Vorstellung finden wir sehr schön bei Kant formuliert. Kant zufolge unterscheidet sich das Recht, die juridische Gesetzgebung, von der ethischen dadurch, daß sie ,eine andere Triebfeder, als die Idee der Pflicht selbst, zuläßt", und diese andere Triebfeder muß Kant zufolge zwangsläufig den Charakter des Zwanges, der Sanktion haben. In Kants Worten: Sie muß „von den pathologischen Bestimmungsgründen der Willkür der Neigungen und Abneigungen und unter diesen von denen der letzteren Art hergenommen sein ..., weil es eine Gesetzgebung, welche nötigend, nicht eine Anlockung, die einladend ist, sein soll. ${ }^{“{ }^{6}}$ Dies ist die Vorstellung, die sich die meisten Laien auch heute noch von der Wirkungsweise des Rechts machen und die gerade für die im Alltagsbewußtsein am ehesten präsenten Rechtsbereiche wie vor allem das Strafrecht ja auch noch weitgehend zutrifft: daß das Recht die mit ihm verfolgten Zielvorstellungen stets unmittelbar in an die Individuen gerichtete sanktionsbewehrte Ge- und Verbote umsetze. Im Falle einer auf $35 \%$ ige Energieeinsparung gerichteten Zielvorstellung liefe dieses Modell etwa auf eine jedermann treffende sanktionsbewehrte Pflicht zu entsprechender Senkung des individuellen Verbrauchs an fossilen Energieträgern hinaus, und dies wäre, ganz abgesehen von allen Problemen der Operationalisierung einer solchen Pflicht, in der Tat eine auch unter Freiheitsgesichtspunkten unerfreuliche Perspektive.

Im Gegensatz zu Kant wissen wir aber heute, und sehen auch kein Problem, sondern im Gegenteil nur Vorteile darin, daß Recht vor allem in der Verfolgung von Zielvorstellungen, die komplexe, sehr unterschiedliche Anpassungsleistungen unterschiedlicher gesellschaftli-

56 Kant (Fn. 16), A 15. 
cher Akteure erfordern, auch ganz anders als auf diese unmittelbar zielbezogen ge- oder verbietende Weise eingesetzt werden kann. Am Beispiel des Energieverbrauchs: Auf dessen Senkung kann das Recht nicht nur dadurch hinwirken, daß es individuelle Energieverbräuche bei Strafe rationiert. Es gibt viele andere Möglichkeiten. Das Steuerrecht kann Vergünstigungen für den Einsatz energiesparender Heizungstechniken gewähren, den Einsatz von Solarenergie fördern und vorhandene energiepolitisch nicht sinnvolle Steuerbegünstigungen (wie z.B. die Steuerbefreiung für Flugbenzin) aufheben. Fossile Energieträger können abgabenrechtlich verteuert und damit Anreize für einen insgesamt geringeren Energieverbrauch unter weitgehender Wahrung individueller Freiheiten der Energienutzung geschaffen werden. Rechtsvorschriften können Hersteller und Händler zur Angabe und Auszeichnung des Energieverbrauchs von Geräten verpflichten, um auf diese Weise Käufern die Betätigung einer Präferenz für wenig Energie verbrauchende Geräte zu ermöglichen, und so weiter.

Individuelle Verhaltensfreiheiten und Spielräume für gesellschaftliche Anpassungsprozesse bleiben unter rechtlichen Regelungen dieser Art sehr viel besser gewahrt als unter der rigiden, um nicht zu sagen fanatischen Moral des individuellen Verzichts, die benötigt würde, wenn man einmal kontrafaktisch unterstellt, daß Energieverbrauchsreduktionen in der erforderlichen Größenordnung überhaupt als Folge bloßer Veränderungen der Umweltmoral erreichbar wären.

\section{Schluß}

Abschließend möchte ich, um Mißverständnissen vorzubeugen, hervorheben, was mit den vorangegangenen Ausführungen nicht behauptet werden sollte:

Ich behaupte nicht, daß unser geltendes Umweltrecht und sonstiges umweltbezogenes Recht in allen Einzelheiten sakrosankt sein sollte, und daß es nicht hier oder dort Regelungen enthalten mag, die überflüssig sind oder einfacher sein könnten. Ich behaupte aber, daß wir für einen wirksamen Umweltschutz insgesamt nicht weniger, sondern 
mehr umweltbezogenes Recht benötigen, und daß die aktuelle umweltpolitische Debatte, die der Sache nach darauf zielt, bislang rechtlich Geregeltes und administrativ Überwachtes wieder mehr der individuellen Moral zu überlassen, auf falschen Vorstellungen über das richtige Verhältnis von Recht und Moral im Umweltschutz beruht.

Ich hoffe auch, deutlich gemacht zu haben, daß mein Plädoyer für die Unentbehrlichkeit und Ausbaubedürftigkeit rechtlicher Instrumente für den Umweltschutz und die zugrundeliegende Überzeugung, daß allein mit Moral hier nicht viel auszurichten ist, die Bedeutung moralischen Verhaltens in Umweltangelegenheiten in keiner Weise herabsetzen will. Es ging mir im Gegenteil gerade darum, deutlich zu machen, daß Recht und Moral, in Umweltangelegenheiten wie auch sonst, gerade nicht gegeneinander ausgespielt werden dürfen, als handele es sich um konkurrierende Lenkungspotentiale, von denen immer nur entweder das eine oder das andere, oder eines nur auf Kosten des anderen, zur Geltung gebracht werden kann. 


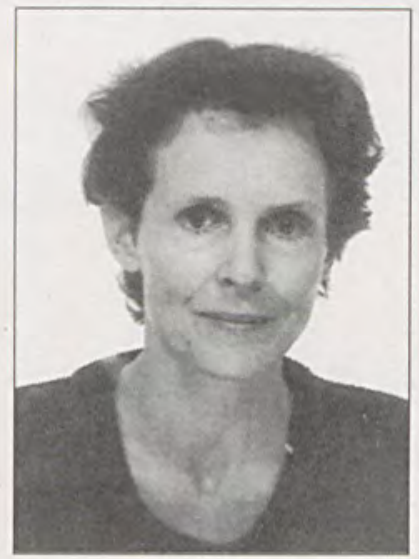

\section{Gertrude Lübbe-Wolff}

1953 Geboren in Weitensfeld/Kärnten

1969 -1975 Studium der Rechtswissenschaft in Bielefeld, Freiburg und an der Havard Law School (1. und 2. Staatsexamen 1974 und 1977)

1980 Promotion in Freiburg

1987 Habilitation an der Universität Bielefeld (Öffentliches Recht, Verfassungsgeschichte der Neuzeit und Rechtsphilosophie)

1988 - 1992 Leiterin des Umweltamtes der Stadt Bielefeld

seit 1992 Professorin für Öffentliches Recht an der Universität Bielefeld. Funktionen u.a.: Geschäftsführende Direktorin des Zentrums für interdisziplinäre Forschung der Universität Bielefeld; Stellv. Wahlmitglied des Verfassungsgerichtshofes für das Land Nordrhein-Westfalen; Mitglied des Vorstandes der Deutschen Sektion der internationalen Vereinigung für Rechts- und Sozialphilosophie e.V.; 1998/99 wissenschaftliche Leitung der Forschungsgruppe „Rationale Umweltpolitik - Rationales Umweltrecht" am Zentrum für Interdisziplinäre Forschung der Universität Bielefeld

\section{Veröffentlichungen:}

Rechtsfolgen und Realfolgen - Welche Rolle können Folgenerwägungen in der juristischen Regel- und Begriffsbildung spielen?, 1981.

Die Grundrechte als Eingriffsabwehrrechte, 1988.

Grundwasserbelastungen durch CKW - Rechtsfragen der Ermittlung und Sanierung, 1991. Modernisierung des Umweltordnungsrechts, 1996.

Umweltschutz durch kommunales Satzungsrecht (Hrsg.), 2. Aufl. 1997.

Der Vollzug des europäischen Umweltrechts (Hrsg.), 1996.

Zahlreiche Aufsätze zum Staats- und Verwaltungsrecht, zur Verfassungsgeschichte und zur Rechtsphilosophie. 


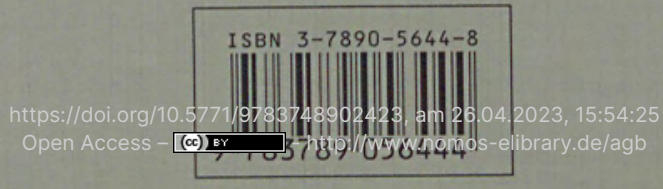

